\title{
Diffusion of large-scale magnetic fields by reconnection in MHD turbulence
}

\author{
R. Santos-Lima, ${ }^{1 \star}$ G. Guerrero, ${ }^{2} \dagger$ E. M. de Gouveia Dal Pino, ${ }^{1} \ddagger$ A. Lazarian ${ }^{3,4} \S$ \\ ${ }^{1}$ Instituto de Astronomia, Geofísica e Ciências Atmosféricas, Universidade de São Paulo, R. do Matão, 1226, São Paulo, SP 05508-090, Brazil \\ ${ }^{2}$ Physics Department, Universidade Federal de Minas Gerais, Av. Antonio Carlos, 6627, Belo Horizonte, MG, Brazil, 31270-901 \\ ${ }^{3}$ Department of Astronomy, University of Wisconsin, 475 North Charter Street, Madison, WI 53706, USA \\ ${ }^{4}$ Center for Computation Astrophysics, Flatiron Institute, 162 5th Ave, New York, NY 10010
}

Last updated 2015 May 22; in original form 2013 September 5

\begin{abstract}
The rate of magnetic field diffusion plays an essential role in several astrophysical plasma processes. It has been demonstrated that the omnipresent turbulence in astrophysical media induces fast magnetic reconnection, which consequently leads to large-scale magnetic flux diffusion at a rate independent of the plasma microphysics. This process is called "reconnection diffusion" (RD) and allows for the diffusion of fields which are dynamically important. The current theory describing RD is based on incompressible magnetohydrodynamic (MHD) turbulence. In this work, we have tested quantitatively the predictions of the RD theory when magnetic forces are dominant in the turbulence dynamics (Alfvénic Mach number $M_{A}<1$ ). We employed the PENCIL CODE to perform numerical simulations of forced MHD turbulence, extracting the values of the diffusion coefficient $\eta_{R D}$ using the Test-Field method. Our results are consistent with the RD theory $\left(\eta_{R D} \sim M_{A}^{3}\right.$ for $\left.M_{A}<1\right)$ when turbulence approaches the incompressible limit (sonic Mach number $M_{S} \lesssim 0.02$ ), while for larger $M_{S}$ the diffusion is faster $\left(\eta_{R D} \sim M_{A}^{2}\right)$. This work shows for the first time simulations of compressible MHD turbulence with the suppression of the cascade in the direction parallel to the mean magnetic field, which is consistent with incompressible weak turbulence theory. We also verified that in our simulations the energy cascading time does not follow the scaling with $M_{A}$ predicted for the weak regime, in contradiction with the RD theory assumption. Our results generally support and expand the RD theory predictions.
\end{abstract}

Key words: magnetic fields - magnetic reconnection - (magnetohydrodynamics) MHD - turbulence - methods: numerical — stars: formation

\section{INTRODUCTION}

One of the most employed and well known concepts in magnetohydrodynamic (MHD) theory is the magnetic "frozenin" condition introduced by Alfvén. When the time scales for Ohmic dissipation of the magnetic fields are much larger than the typical dynamical time scales of the flow (the dimensionless parameter characterizing the ratio between these two time scales being given by the magnetic Reynolds number $R_{M}=U L / \eta$, with $U$ and $L$ the characteristic velocity and scale of the flow, and $\eta$ the magnetic diffusivity provided by the Ohmic dissipation), one can adopt the ideal MHD approximation. It consists in neglecting the resistive

\footnotetext{
* Contact e-mail: reinaldo.lima@iag.usp.br

† Contact e-mail: guerrero@fisica.ufmg.br

‡ Contact e-mail: dalpino@iag.usp.br

$\S$ Contact e-mail: lazarian@astro.wisc.edu
}

term in the magnetic induction equation. In this limit, it can be demonstrated that the magnetic flux across a Lagrangian fluid element is conserved, that is, the magnetic field is perfectly advected by the fluid motions in the direction normal to the field lines.

The ideal MHD description (and consequently, the frozen-in condition) is usually thought to be a good approximation for most astrophysical plasmas, which have in general huge values of $R_{M}$. Nevertheless, the frozen in condition when applied, for instance, to star formation regions gives rise to several problems due to observational and theoretical requirements for diffusive magnetic flux transport through the plasma (e.g. Santos-Lima, et al. 2010; Santos-Lima, de Gouveia Dal Pino \& Lazarian 2012, 2013; Leão, et al. 2013; González-Casanova, Lazarian \& Santos-Lima 2016). Ambipolar diffusion is usually invoked for breaking the frozen-in 


\section{Santos-Lima, Guerrero, de Gouveia Dal Pino $\&$ Lazarian}

condition and solving the problem of the magnetic flux transport during star formation (see for example Shu 1983; Nishi, Nakano, \& Umebayashi 1991; Ciolek \& Mouschovias 1993; Shu et al. 1994; Tassis \& Mouschovias 2005). However, several studies revealed weaknesses in this solution (Shu, et al. 2006; Crutcher, Hakobian \& Troland 2009; Krasnopolsky, Li \& Shang 2010, 2011; Li, Krasnopolsky \& Shang 2011). A potential solution for this problem in the framework of protoplanetary disk formation has been proposed by Machida, Inutsuka \& Matsumoto (2007, 2009) based on laminar MHD simulations combined with local Ohmic resistivity (see also recent studies on the role of the ambipolar diffusion during protostellar disk formation Wurster \& Li 2018; Guillet et al. 2020; Marchand et al. 2020; Zhao et al. 2020).

We believe that the limitations of the approach that is described above is that the effects of ubiquitous astrophysical turbulence are disregarded in the aforementioned studies. The diffuse interstellar medium and molecular clouds are turbulent. There are overwhelming observational evidences that support this claim through the measurements of power spectrum of densities in diffuse ISM (see Armstrong, Rickett \& Spangler 1995; Chepurnov \& Lazarian 2010), broadening of the molecular lines (see Larson 1981), statistics of velocities (see Lazarian \& Pogosyan 2000; Lazarian 2009 and references therein, Padoan, et al. 2009; Chepurnov, et al. 2010, 2015; Kandel, Lazarian \& Pogosyan 2017; Utomo, Blitz \& Falgarone 2019; Wolleben, et al. 2019; $\mathrm{Xu}$ 2020; Yuen, et al. 2019), variations of the Faraday rotation (Haverkorn, et al. 2008; Xu \& Zhang 2016), and power spectrum of synchrotron fluctuations (Chepurnov 1998; Cho \& Lazarian 2002, 2010). The reviews describing the molecular cloud turbulence are presented in McKee \& Ostriker (2007); Mac Low \& Klessen (2004, see also Elmegreen \& Scalo 2004).

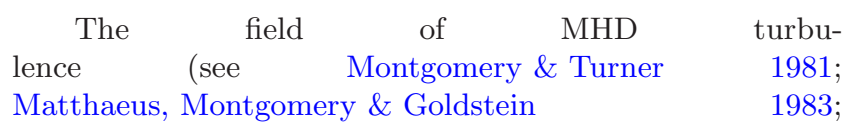

Shebalin, Matthaeus \& Montgomery 1983; Higdon 1984) has seen a rapid progress due to both, to Solar wind measurements (see Tu \& Marsch 1995; Goldstein, et al. 1995; Bruno \& Carbone 2013 for a review), theoretical (see Goldreich \& Sridhar 1995; Lazarian \& Vishniac 1999; Lithwick \& Goldreich 2001; Cho \& Lazarian 2002; Eyink, Lazarian \& Vishniac 2011) and numerical progress (Cho \& Vishniac 2000; Maron \& Goldreich 2001; Cho, Lazarian \& Vishniac 2002; Cho \& Lazarian 2003; Kowal \& Lazarian 2010; Federrath, et al. 2010; Beresnyak 2014; see also a recent book by Beresnyak \& Lazarian 2019).

As we discuss later, the subject of MHD turbulence is closely related to the processes of magnetic reconnection in turbulent fluid. The model of turbulent reconnection in Lazarian \& Vishniac (1999) predicts the failure of the traditional flux freezing in highly conducting turbulent fluids. This process does not depend on the rate of ambipolar diffusion and motivated a number of our earlier studies (Lazarian 2005, 2011; Santos-Lima, et al. 2010; Santos-Lima, de Gouveia Dal Pino \& Lazarian 2012, 2013; González-Casanova, Lazarian \& Santos-Lima 2016) that consider the processes of magnetic flux transport in turbulent fluids.

The process by which the topology of the magnetic field changes depends on whether the fluid is in a laminar or turbulent state. In the presence of turbulence, the motions of the ionized gas produce tangling and wandering of the magnetic field lines which give origin to several micro-sites of magnetic reconnection. This process is independent on how small is the Ohmic resistivity which is always present in any real plasma. These reconnection micro-sites are continuously formed and spread all over the turbulent plasma volume. As a consequence, the field lines topology can be modified, and large-scale magnetic flux can be transported through the gas, implying that the flux freezing concept is seriously altered (Lazarian 2005; Eyink, Lazarian \& Vishniac 2011). The speed at which the magnetic flux is transported in such conditions is independent of the electric resistivity of the plasma, or the degree of its ionization but is regulated by the turbulence parameters, as predicted in the theory of fast magnetic reconnection introduced by Lazarian \& Vishniac (1999). This theory was tested numerically in Kowal, et al. (2009, 2017, 2020). A convincing quantitative numerical study proving that turbulent reconnection violates flux freezing in MHD turbulence is presented in Eyink, et al. (2013). An extensive body of evidence in favor of turbulence reconnection has been collected by now and we refer the reader to Lazarian, et al. (2020) where the modern state of the turbulent reconnection theory and a description of its tests with solar wind as well as numerical testing with different codes is reviewed.

The concept of magnetic diffusion via turbulent reconnection - Reconnection Diffusion (henceforth RD) - is distinct from the concept of standard turbulent mixing. The latter is based on the idea that the field lines are mixed passively by the turbulent eddies, without taking into account the effects of the magnetic field on the turbulent cascade. RD on the other hand, covers the interesting situation in which the magnetic forces are dynamically important (e.g. in the late stages of star formation), and relies on the fact that the fast reconnection induced by the MHD turbulence is independent of the value of the electric resistivity of the plasma. For many of the astrophysical applications it is important that the RD is not altered by the effects of ambipolar drift on the scales where turbulence exists.

This RD theory predicts that the diffusion coefficient $\eta_{R D}$ for large-scale magnetic fluxes (i.e., scales larger than the injection or forcing scale of the turbulence) depends on the turbulence parameters as follows. In the case of superAlfvénic turbulence (that is, when the Alfvénic Mach number, $M_{A}=U_{\text {turb }} / v_{A}\left[U_{\text {turb }}\right.$ is the turbulent velocity and $v_{A}$ is the local Alfvén velocity] is larger than one), it coincides with the standard turbulent mixing coefficient,

$\eta_{\mathrm{RD}} \sim L_{\text {turb }} U_{\text {turb }}$

where $L_{\text {turb }}$ and $U_{\text {turb }}$ are the length and the velocity of the turbulence at the injection scale, respectively. On the other hand, in the regime of sub-Alfvénic turbulence $\left(M_{A}<1\right)$, this value is reduced by a factor proportional to the third power of $M_{A}$ (Lazarian 2006, 2011):

$\eta_{\mathrm{RD}} \sim L_{\text {turb }} U_{\text {turb }} M_{A}^{3}$.

Therefore, according to the RD theory, the efficiency of 
the magnetic flux transport strongly depends on the local turbulence regime. In the context of star formation, the turbulence parameters in scales below sub-parsec (down to dozens of A.U.) can be difficult to infer with precision. Nonetheless, we reinforce that RD process is always present at some level during all the star formation process. It is a natural consequence of the ubiquitous presence of turbulence in astrophysical environments (see for example Krumholz \& McKee 2005; Ballesteros-Paredes et al. 2007; Hennebelle \& Chabrier 2011; Padoan \& Nordlund 2011; Federrath \& Klessen 2012, 2013; Hull, et al. 2017). In previous work (Santos-Lima, et al. 2010; Santos-Lima, de Gouveia Dal Pino \& Lazarian 2012, 2013; Leão, et al. 2013; González-Casanova, Lazarian \& Santos-Lima 2016), we investigated numerically the removal of magnetic flux from collapsing turbulent molecular clouds and protostellar disks, considering an "ideal" MHD approach (i.e., the microscopic magnetic dissipation term was not considered explicitly in the induction equation, although an effective value is always present due to the numerical discretization of the equations). We found that the magnetic flux removal by $\mathrm{RD}$ is efficient in these systems, and helps the gravitational collapse of the structures (see also Myers, et al. 2013). However, these works focused mostly on the super and trans-Alfvénic regimes of the turbulence where the reconnection diffusion coefficient is controlled by Eq. (1). The aim of this work is to test quantitatively the prediction of $E q$. (2) by using three-dimensional (3D) MHD simulations. It is also the first attempt to generate simulations of stationary weak MHD turbulence (the cenario invoked by the $R D$ theory) in the presence of finite compressibility, which is more realistic for astrophysical environments.

This work in organized as follows. In $\S 2$ we present the main predictions of the RD theory. The numerical methods and setup for the numerical simulations of this study are described in $\S 3$. The results are presented and discussed in $\S 4$ and $\S 5$, respectively. Our major findings are finally summarized in $\S 6$.

\section{BASICS OF RECONNECTION DIFFUSION THEORY}

To understand the process of reconnection diffusion we present here some basic facts of MHD turbulence theory. For simplicity, we consider only the case of incompressible MHD turbulence.

Traditionally, Alfvénic turbulence is described in terms of non-linearly interacting wave packets in Fourier space (Iroshnikov 1963; Kraichnan 1965). The non-linear cascading rate is given in this case by

$\tau_{\text {casc }}^{-1} \approx \frac{\left(\delta u_{\ell} / \ell_{\perp}\right)^{2}}{\omega_{A}}$

where the angular frequency, $\omega=V_{A} / \ell_{\|}$, and $\ell_{\|}$is the parallel wavelength. If the injection velocity $U_{L}$ is less than the Alfvén velocity, the cascade is evolving decreasing only perpendicular wavelengths $\ell_{\perp}$. This is the regime of weak turbulence with the parallel scale being all the time equal to the injection scale and $\delta u_{\ell} \sim \ell_{\perp}^{1 / 2}$ (Lazarian \& Vishniac 1999 [LV99 hereafter]; Galtier, et al. 2000). However the weak cascade changes its nature at a small scale. Below we explain the nature of this change.

The theory of strong MHD turbulence was formulated by Goldreich \& Sridhar (1995, henceforth GS95) for transAlfvénic turbulence, i.e. $U_{L}=V_{A}$. For this turbulence GS95 formulated the condition of the critical balance that relates the scale of parallel and perpendicular motions, namely, $\delta u_{\ell} / \ell_{\perp} \approx V_{A} / \ell_{\|}$. This condition means that the parallel scale should change with the decrease of the perpendicular scale.

The transfer to the strong MHD turbulence regime can happen if the turbulence is injected at $U_{L}<V_{A}$ and is weak at its origin. The key to that is the increase of the strength of non-linear interactions with the decrease of perpendicular scale. The transfer to the strong turbulence regime changes the nature of the turbulent motions. In fact, due to fast turbulent reconnection predicted in LV99 the turbulent motions become similar to the hydrodynamic eddies that mix magnetic field perpendicular to the direction of magnetic field. For this eddies it is natural to assume that $\delta u_{\ell} / \ell \sim V_{A} / \ell_{\|}$, i.e., that the rate of turbulent mixing motions is equal to the rate of perturbation propagating along the magnetic field. The difference between the original critical balance in GS95 and the above relation between the perpendicular scale $\ell$ and the parallel scale $\ell_{\|}$is that both scales are measured in terms of the magnetic field of the eddies, i.e. local magnetic field, compared to the wavenumbers $k_{\|}$and $k_{\perp}$ that are given in the global mean magnetic field reference system. The concept of local eddies mixing up magnetic field is an essential component for the understanding of the RD process. Note that numerical research does indicate that the critical balance relations are valid only in the local system of reference (Cho \& Vishniac 2000; Maron \& Goldreich 2001; Cho, Lazarian \& Vishniac 2002).

The generalization of GS95 theory for subAlfvénic turbulence provided in LV99 as well as the analogy between the eddy turbulence in MHD and ordinary hydrodynamic turbulence helps to understand the nature of the RD. The predictions for $\mathrm{RD}$ when turbulence is in the sub-Alfvénic regime (Lazarian 2005, 2011) can be recovered from the statistical calculations presented in Eyink, Lazarian \& Vishniac (2011), and are summarized bellow.

The magnetic field is assumed to be diffused by turbulence at a rate similar to the diffusion of trace particles in the direction perpendicular to the (locally) uniform magnetic field. A simple statistical analysis can show that the perpendicular diffusivity of trace particles due to the turbulent velocity field $\delta \mathbf{u}$ is given by:

$D_{\perp}=\int_{-\infty}^{\infty} d t\left\langle\delta \mathbf{u}_{\perp}^{\prime}(0) \cdot \delta \mathbf{u}_{\perp}^{\prime}(t)\right\rangle$

where $\delta \mathbf{u}^{\prime}(t)$ is the turbulent velocity at the position of the particle at time $t$, and the angle brackets, $\langle\cdot\rangle$, denote an ensemble average over all the particles, which are assumed to be distributed randomly in space. Considering turbulence composed by Alfvén waves with random phases and turbulence with a single scale, $\ell$, perpendicular to the field lines (and corresponding parallel scale, $\ell_{\|}$). The expression inside the last integral is

$\left\langle\delta \mathbf{u}_{\ell, \perp}^{\prime}(0) \cdot \delta \mathbf{u}_{\ell, \perp}^{\prime}(t)\right\rangle \sim \delta u_{\ell}^{2} \Re\left\{\exp \left(i \omega_{A, \ell_{\|}} t-|t| / \tau_{\mathrm{dec}}\right)\right\}$,

where $\delta u_{\ell}$ is the perpendicular turbulent velocity at scale $\ell$, 


\section{Santos-Lima, Guerrero, de Gouveia Dal Pino $\&$ Lazarian}

$\omega_{A, \ell_{\|}} \sim V_{A} / \ell_{\|}$is the local Alfvén frequency, and $\tau_{\text {dec }}$ is the time-scale for decorrelation of the velocity eddies (the correlation is simply assumed to decay exponentially in time). This leads to

$D_{\perp}(\ell) \sim \delta u_{\ell}^{2} \frac{\tau_{\mathrm{dec}}}{\left(\omega_{A, \ell_{\|}} \tau_{\mathrm{dec}}\right)^{2}+1}$.

We expect that motions at the largest scales produce the dominant diffusivity. We will assume isotropy at the injection scale, that is, $\ell_{\|} \sim \ell$.

When the time-scale for the microscopic diffusivity is larger than the dynamical time-scales of the system (which is the case for most astrophysical environments), the decorrelation time for the velocity eddies at the injection scale should be of the order of the energy cascade time for these eddies. In the regime of weak turbulence (i.e., when the energy cascading time is much longer than the linear wave time $\tau_{\mathrm{w}} \sim$ $\left.\omega_{A, \ell_{\|}}^{-1}\right)$, we have $\tau_{\text {casc }} \sim\left(\ell / \delta u_{\ell}\right) M_{A}^{-1}$ (Lazarian \& Vishniac 1999; Galtier, et al. 2000). Substituting this value in Eq. (6), we obtain the RD prediction for the magnetic diffusivity $\eta_{\mathrm{RD}}$ (Lazarian 2005):

$\eta_{\mathrm{RD}} \sim D_{\perp} \sim \ell \delta u_{\ell} \min \left(1, M_{A}^{3}\right)$.

In situations where the energy cascading time at the injection scale is larger than the molecular or numerical viscous time (implying a low effective Reynold's number), the decorrelation time of the velocity, $\tau_{\text {dec }}$, can be more closely related to the dissipation time, $\tau_{\text {diss }} \sim \ell^{2} / \nu$, where $\nu$ is the molecular viscosity. The magnetic field diffusion driven by turbulence in this case will depend on the microscopic diffusion and, if $\tau_{\text {dec }} \sim \tau_{\text {diss }}$, then the diffusivity becomes dependent on the molecular viscosity, $\nu$.

In our discussion we considered only one component of MHD turbulence, namely, Alfvén modes and disregarded the slow and fast modes (see Cho \& Lazarian 2003). This is due to the fact that Alfvén modes are the most important in mixing the medium.

\section{NUMERICAL MODELS}

In order to test the dependency of the magnetic diffusion coefficient, $\eta_{\mathrm{RD}}$, with the Alfvénic Mach number, $M_{A}$, of the turbulence in the sub-Alfvénic regime, we employed threedimensional MHD numerical simulations of forced turbulence in a Cartesian domain, in the presence of an external uniform magnetic field of intensity $B_{0}$ in the $x$-direction. We used the PEncIL Code ${ }^{1}$ for numerically solving the set of compressible, isothermal, MHD equations:

$\frac{D \ln \rho}{D t}=-\nabla \cdot \mathbf{u}$

$\frac{D \mathbf{u}}{D t}=-c_{s}^{2} \nabla \ln \rho+\frac{1}{\rho} \mathbf{J} \times\left(\mathbf{B}_{\mathbf{0}}+\mathbf{B}\right)+\nu_{3} \nabla^{6} \mathbf{u}+\mathbf{f}$,

$\frac{\partial \mathbf{A}}{\partial t}=\mathbf{u} \times\left(\mathbf{B}_{\mathbf{0}}+\mathbf{B}\right)+\eta_{3} \nabla^{6} \mathbf{A}$,

1 http://pencil-code.googlecode.com/ where $D / D t=\partial / \partial t+\mathbf{u} \cdot \nabla$ is the lagrangian derivative, $\mathbf{A}$ is the magnetic potential vector, $\mathbf{B}=\nabla \times \mathbf{A}$ is the magnetic field generated by the internal currents, $\mathbf{J}=\nabla \times \mathbf{B} / \mu_{0}$ is the current density, $\mu_{0}$ is the magnetic permeability, $\nu_{3}$ and $\eta_{3}$ are, respectively, the coefficients of hyper-viscosity and magnetic hyper-diffusivity, $c_{s}$ is the isothermal sound speed, $\mathbf{u}$ is the velocity, $\rho$ is the density, and $\mathbf{f}$ represents the force responsible for the turbulence injection. We use the hyper-viscosity and magnetic hyper-diffusivity schemes with the aim of obtaining a turbulent spectra with an extension as large as possible for each considered resolution (Borue \& Orszag 1995; Haugen \& Brandenburg 2004). The value of the coefficients were minimized such that numerical stability is guaranteed.

We have also performed simulations without the hyper-viscosity and the magnetic hyper-diffusivity. In these cases, we employed the usual viscosity and Ohmic resistivity, namely, the terms $\nu_{3} \nabla^{6} \mathbf{u}$ in eq. 9 and $\eta_{3} \nabla^{6} \mathbf{A}$ in eq. 10 are replaced by $\eta_{1}\left\{\nabla^{2} \mathbf{u}+(1 / 3) \nabla \nabla \cdot \mathbf{u}+2 \mathbf{S} \cdot \nabla \ln \rho\right\} \quad$ and $\eta_{1} \nabla^{2} \mathbf{A}$, respectively, where $\nu_{1}$ is the constant viscosity, $\eta_{1}$ is the magnetic diffusivity, and $\mathbf{S}$ is the rate of strain tensor given by $S_{i j}=\left\{(1 / 2)\left(\partial u_{i} / \partial x_{j}+\partial u_{j} / \partial x_{i}\right)-(1 / 3) \delta_{i j} \nabla \cdot \mathbf{u}\right\}$. These models are explicitly mentioned in the text whenever they appear.

The turbulence is constantly forced by the increment of the velocity field with a spectrum of Fourier modes. These modes are purely solenoidal, and the phases are randomly changed at every iteration during the numerical integration. The turbulence is, therefore, nearly statistically homogeneous, non-helical, and delta correlated in time. In $\S 3.1$ we provide more details about the spectrum of the excited velocity modes.

The value of the diffusion coefficient, $\eta_{\mathrm{RD}}$, is extracted from the simulations through the Test-Field method. It employs a set of passive test magnetic fields in order to calculate unambiguously the coefficients of the turbulent mean-fields (including the diffusion tensor; see the Appendix A and more details in Brandenburg \& Subramanian 2005; Schrinner, et al. 2007; Brandenburg, et al. 2010).

\subsection{Setup and parameters}

The Reconnection Diffusion theory $(\S 2)$ is formulated in the incompressible limit, assuming that when sub-Alfvénic turbulence is forced isotropically in the presence of an uniform magnetic field (at least locally), it will develop a cascade in the regime predicted by the weak turbulence theory. Therefore, we restricted our simulations setup and parameters to the subsonic regime (yet using finite sound speed $c_{s}$ in the simulations) and favoring conditions under which weak turbulence could develop.

Table 1 lists the parameters employed in the simulations presented in the next Section. For each set of simulations (identified by its name in the first column of Table 1), we spanned a range of values of $M_{A}$ by changing the magnetic field strength $B_{0}$ and keeping the rms value of the turbulence velocity, $v_{\text {rms }}$, constant, near to a fixed reference value, $v_{0}$. We do not control directly $v_{\text {rms }}$ in our simulations, but instead, the amplitude of the forcing.

The turbulent diffusivity is controlled by the largest scale motions of the system (the motions at the injection 
Table 1. Runs parameters

\begin{tabular}{|c|c|c|c|c|c|c|c|c|c|c|}
\hline runs set & $L_{\|} \mathrm{x} L_{\perp}$ & res. & $M_{S}^{\mathrm{a}}$ & $M_{A}^{\mathrm{b}}$ & $v_{r m s} / v_{0}$ & forcing & $k_{\|} L / 2 \pi$ & $k_{\perp} L / 2 \pi^{\mathrm{c}}$ & {$\left[\tilde{t}_{0}, \tilde{t}_{1}\right]^{\mathrm{d}}$} & $\tilde{\nu}_{3}, \tilde{\eta}_{3}{ }^{\mathrm{e}}$ \\
\hline $16 \mathrm{~L}-\mathrm{Ms} 0.32-\mathrm{A}$ & $16 \mathrm{Lx} 1 \mathrm{~L}$ & $2048,128^{2}$ & 0.32 & $\begin{array}{l}0.8,0.57 \\
0.4,0.2 \\
0.1\end{array}$ & $\begin{array}{l}1.10,1.18 \\
1.18,1.22 \\
1.33\end{array}$ & A & {$[0,4]$} & {$[3,4]$} & {$[3,8]$} & $1.8 \times 10^{-9}$ \\
\hline $16 \mathrm{~L}-\mathrm{Ms} 0.08$ & $16 \mathrm{Lx} 1 \mathrm{~L}$ & $2048,128^{2}$ & 0.08 & $\begin{array}{l}0.8,0.4, \\
0.28,0.2 \\
0.1\end{array}$ & $\begin{array}{l}1.09,1.17, \\
1.11,1.10 \\
1.11\end{array}$ & $\mathrm{~A}$ & {$[0,4]$} & {$[3,4]$} & $\begin{array}{l}{[3,8],[4,9]} \\
{[7,12],[7,12]} \\
{[9,14]}\end{array}$ & $1.8 \times 10^{-9}$ \\
\hline $16 \mathrm{~L}-\mathrm{Ms} 0.02-\mathrm{A}$ & $16 \mathrm{Lx} 1 \mathrm{~L}$ & $2048,128^{2}$ & 0.02 & $\begin{array}{l}0.8,0.4 \\
0.28,0.2 \\
0.1\end{array}$ & $\begin{array}{l}1.08,1.17 \\
1.27,1.04 \\
1.04\end{array}$ & $\mathrm{~A}$ & {$[0,4]$} & {$[3,4]$} & $\begin{array}{l}{[3,8],[4,9]} \\
{[4,9],[13,18]} \\
{[15,20]}\end{array}$ & $1.8 \times 10^{-9}$ \\
\hline 8L-Ms0.02-A & $8 \mathrm{Lx} 1 \mathrm{~L}$ & $1024,128^{2}$ & 0.02 & $\begin{array}{l}0.8,0.4 \\
0.2,0.1\end{array}$ & $\begin{array}{l}1.09,1.17 \\
0.99,1.01\end{array}$ & $\mathrm{~A}$ & {$[0,4]$} & {$[3,4]$} & $\begin{array}{l}{[3,8],[4,9]} \\
{[13,18],[15,20]}\end{array}$ & $1.8 \times 10^{-9}$ \\
\hline $4 \mathrm{~L}-\mathrm{Ms} 0.02-\mathrm{A}$ & $4 \mathrm{Lx} 1 \mathrm{~L}$ & $512,128^{2}$ & 0.02 & $\begin{array}{l}0.8,0.4 \\
0.2,0.1\end{array}$ & $\begin{array}{l}1.10,1.19 \\
0.94,1.01\end{array}$ & $\mathrm{~A}$ & {$[0,4]$} & {$[3,4]$} & $\begin{array}{l}{[3,8],[4,9]} \\
{[13,18],[15,20]}\end{array}$ & $1.8 \times 10^{-9}$ \\
\hline $1 \mathrm{~L}-\mathrm{Ms} 0.02-\mathrm{A}$ & $1 \mathrm{Lx} 1 \mathrm{~L}$ & $128,128^{2}$ & 0.02 & $\begin{array}{l}0.8,0.4 \\
0.2,0.1\end{array}$ & $\begin{array}{l}1.22,1.19 \\
1.04,1.11\end{array}$ & A & {$[0,4]$} & {$[3,4]$} & $\begin{array}{l}{[3,8],[4,9]} \\
{[7,12],[7,12]}\end{array}$ & $1.8 \times 10^{-9}$ \\
\hline $8 \mathrm{~L}-\mathrm{Ms} 0.02-\mathrm{Ab}$ & $8 \mathrm{Lx} 1 \mathrm{~L}$ & $1024,128^{2}$ & 0.02 & $\begin{array}{l}0.8,0.4 \\
0.2,0.1\end{array}$ & $\begin{array}{l}1.07,1.10 \\
1.09,0.93\end{array}$ & $\mathrm{Ab}$ & - & {$[4,5]$} & $\begin{array}{l}{[3,8],[4,9]} \\
{[10,15],[15,20]}\end{array}$ & $1.8 \times 10^{-9}$ \\
\hline 8L-Ms0.02-I & $8 \mathrm{Lx} 1 \mathrm{~L}$ & $1024,128^{2}$ & 0.02 & $\begin{array}{l}0.8,0.4 \\
0.2,0.1\end{array}$ & $\begin{array}{l}1.07,1.11 \\
1.19,1.19\end{array}$ & $\mathrm{I}$ & - & {$[3,4]$} & $\begin{array}{l}{[3,8],[4,9]} \\
{[4,9],[4,9]}\end{array}$ & $1.8 \times 10^{-9}$ \\
\hline 16L-Ms0.02-low-A & $16 \mathrm{Lx} 1 \mathrm{~L}$ & $1024,64^{2}$ & 0.02 & $\begin{array}{l}0.8,0.4 \\
0.2,0.14 \\
0.1,0.05 \\
0.025\end{array}$ & $\begin{array}{l}1.02,1.12, \\
1.31,1.43 \\
1.44,1.40 \\
1.32\end{array}$ & A & {$[0,4]$} & {$[3,4]$} & {$[11,15]$} & $6.2 \times 10^{-8}$ \\
\hline 16L-Ms0.02-low-A-diff2 & $16 \mathrm{Lx} 1 \mathrm{~L}$ & $1024,64^{2}$ & 0.02 & $\begin{array}{l}0.8,0.4 \\
0.2,0.1\end{array}$ & $\begin{array}{l}1.18,1.28 \\
1.37,1.43\end{array}$ & $\mathrm{~A}$ & {$[0,4]$} & {$[3,4]$} & {$[4,8]$} & $\left(1.2 \times 10^{-2}\right)^{\mathrm{f}}$ \\
\hline 16Lx2L-Ms0.02-low-A & $16 \mathrm{Lx} 2 \mathrm{~L}$ & $1024,128^{2}$ & 0.02 & 0.2 & 1.30 & $\mathrm{~A}$ & {$[0,4]$} & {$[3,4]$} & {$[11,15]$} & $6.2 \times 10^{-8}$ \\
\hline 16L-Ms0.02-hi-A & $16 \mathrm{Lx} 1 \mathrm{~L}$ & $2048,256^{2}$ & 0.02 & 0.2 & 0.90 & $\mathrm{~A}$ & {$[0,4]$} & {$[3,4]$} & {$[3,5]^{\mathrm{g}}$} & $\left(5.5 \times 10^{-11}\right)^{\mathrm{h}}$ \\
\hline
\end{tabular}

a $M_{S} \equiv v_{0} / c_{S}$ is the approximate sonic Mach number of the simulations.

b $M_{A} \equiv v_{0} / v_{A, 0}$ is the approximate Alfvénic Mach number of the simulations.

c For the models with forcings 'Ab' and ' $\mathrm{I}$ ', this column shows the absolute values of the vector $\mathbf{k}$ of the forced modes.

$\mathrm{d}\left[\tilde{t}_{0}, \tilde{t}_{1}\right]$ is the time interval used for the averages in time, in units of $\ell_{\perp} / v_{0}$.

e $\tilde{\nu}_{3}, \tilde{\eta}_{3}$ are the hyper-viscosity and hyper-resistivity in units of $\ell_{\perp}^{5} v_{0}$.

${ }^{\mathrm{f}}$ No hyper-viscosity or hyper-resistivity were used in these runs. This column shows the values of $\tilde{\nu}_{1}$ and $\tilde{\eta}_{1}$, the viscosity and magnetic diffusivity, respectively, in units of $\ell_{\perp} v_{0}$. See Section 3 for more details.

g This simulation used as initial condition a previously evolved simulation with resolution $2048,128^{2}$.

${ }^{\mathrm{h}}$ Due to the anisotropic resolution in this simulation, these values of $\tilde{\nu}_{3}$ and $\tilde{\eta}_{3}$ refer to the terms in the hyper-viscosity and hyper-resistivity containing the derivatives in the directions perpendicular to the mean magnetic field. The values of $\tilde{\nu}_{3}$ and $\tilde{\eta}_{3}$ used in the terms containing the derivatives in the parallel direction are $1.8 \times 10^{-9}$.

scale), where the universal laws of the inertial range are not formally valid, and could be affected by the details of the forcing mechanism. At the same time, the forcing can also determine the MHD turbulence regime. Because this work focuses on the subsonic turbulence, we employed forcing schemes purely solenoidal, and with approximately the same coherence length in the directions parallel and perpendicular to the mean magnetic field. The forcing was chosen delta correlated in time for two reasons: first, if the cascading time follows the weak turbulence theory, the correlation of the waves at the injection scale should persist for a time similar to the cascading time, even if the forcing is generating low energy random waves continuously. Second, forcing with time correlation proportional to $M_{A}^{-1}$ (e.g., Alexakis 2011) must induce Alfvén waves with approximately the same decorrelation time, which could determine trivially the diffusion coefficient.

We employed three forcing schemes, which differ in the discrete spectrum of the velocity modes excited. In one of them, all the modes inside a spherical shell in the $\mathbf{k}$-space are excited with the same amplitude. This $\mathbf{k}$-isotropic distribution of amplitudes is the most usual choice in numerical simulations of forced turbulence. The models using this scheme are identified by 'I' in the column "forcing" of Table 1. Alternatively, in order to constrain the parallel and perpendicular injection scales to well defined values, $\ell_{\|}$and $\ell_{\perp}$, respectively, and at the same time avoid the forcing of purely $2 \mathrm{D}$ modes (i.e., with $k_{\|}=0$ ) as well as waves of large wavelength in the direction parallel to the imposed uniform magnetic field, we also used an k-anisotropic scheme (identified by 'A' in Table 1). It forces all the waves with the components $k_{\perp}$ and $k_{\|}$inside a cylindrical shell in the $\mathbf{k}$ space. The amplitude of the spectrum is modulated by a factor $\propto k_{\|}^{2}$. The third forcing scheme we used excites all the modes inside a spherical shell in the $\mathbf{k}$-space, but modulates the amplitudes by a factor $\propto \sin (2 \theta)$, where $\cos \theta=k_{\|} / k$. This last scheme is identified by 'Ab' in Table 1 . The ' $A$ ' and 'Ab' schemes favor distributions with reduced amplitude for 
the wavevectors corresponding to wavelengths far from the fixed injection scale. The forcing scheme 'I', on the other side, generates waves elongated in both parallel and perpendicular directions. We emphasize that, statistically, all the three schemes force velocity fluctuations in an (nearly) isotropic form in the physical space.

The injection scales are indicated in the columns $k_{\|} L / 2 \pi$ and $k_{\perp} L / 2 \pi$, where $\mathrm{L}$ is the shortest side of the domain, perpendicular to the mean uniform magnetic field, in Table 1 (except for the runs set 16Lx2L-Ms0.02-low-A, where this side has length $2 \mathrm{~L}$ ). These values were chosen in order to maximize the turbulence inertial range. Note, however, that although the separation of scales between the largest turbulence eddies and the mode employed in the test fields $\left(k_{\perp, \mathrm{tf}} \mathrm{L} / 2 \pi=k_{z, \mathrm{tf}} \mathrm{L} / 2 \pi=1\right.$; see Appendix A) is still limited.

Unlike real extended astrophysical environments, the finiteness of the computational box introduces effects on the wave turbulence due to insufficient density of large-scale modes represented in the discrete Fourier space. A theoretical constraint on the validity of the classical weak turbulence regime in a finite box domain, which was derived both under the Reduced MHD approximation and the assumption of $k_{\perp} \gg k_{\|}$(Nazarenko 2007), is approximately given by

$\sqrt{\frac{\ell_{\|}}{L_{\|}}} \ll \frac{\delta u_{\ell}}{B_{0}} \frac{\ell_{\|}}{\ell_{\perp}} \ll 1$,

where $\delta u_{\ell}$ is the velocity amplitude corresponding to the mode with scales $\ell_{\|}$and $\ell_{\perp}$. Therefore, to fullfill this condition at the injection scale where $\ell_{\|} \approx \ell_{\perp}$, it is needed to ensure $\sqrt{\ell / L_{\|}} \ll M_{A} \ll 1$.

Another effect due to the finite box size is the 2D "enslaving" of the 3D MHD turbulence if

$\frac{\ell_{\perp}}{L_{\perp}} \sqrt{\frac{\ell_{\|}}{L_{\|}}} \gg \frac{\delta u_{\ell}}{B_{0}} \frac{\ell_{\|}}{\ell_{\perp}}$,

(Nazarenko 2007), which at the injection scales becomes $\left(\ell / L_{\perp}\right) \sqrt{\ell / L_{\|}} \ll M_{A}$. It should be noted that if the leftmost inequality in Eq. (11) is satisfied, then the inequality in Eq. (12) is automatically false $\left(\ell / L_{\perp}<1\right)$.

To evaluate the finite size effects of the computational domain on the RD coefficient we ran simulations with four different domain sizes in the direction parallel to the mean magnetic field ( $x$-direction): $L_{\|}=16 \mathrm{~L}, 8 \mathrm{~L}, 4 \mathrm{~L}$, and $1 \mathrm{~L}$, keeping fixed the domain size perpendicular $\mathrm{L}_{\perp}=1 \mathrm{~L}$. To verify a possible influence of the perpendicular domain size on the $R D$ coefficients, we ran a comparative model with $\mathrm{L}_{\perp}=2 \mathrm{~L}$, keeping $L_{\|}=16 \mathrm{~L}$ (runs set 16Lx2L-Ms0.02-low-A).

In the results presented in the next Section the analysis of the simulations is performed after the turbulence has reached the statistically stationary state. The analyses average quantities between times $\tilde{t}_{0}$ and $\tilde{t}_{1}$ shown in Table 1. Four complete snapshots with equal time separation are extracted from the simulations during this time interval (the only exception is the model $16 \mathrm{Lx} 1 \mathrm{~L}-\mathrm{Ms} 0.02-\mathrm{hi}-\mathrm{A}$, which has only two complete snapshots).

Figure 1 compares the distribution of the velocity modulus on the central xy-plane for three selected runs from Table 1 at time $\tilde{t}_{1}$ (see Table 1 ). The three models differ only by the forcing scheme: A (top), Ab (middle), and I (bottom). All the runs in Figure 1 have $M_{A}=0.4$. Fig- ure 2 depicts the same quantity for simulations that have $M_{A}=0.2$. Observe that the differences between the turbulent structures generated by the different forcings become more pronounced for smaller values of $M_{A}$ (Figure 2). For $M_{A}=0.8$ (not shown here for compactness), the velocity distribution resulting from the three forcing schemes is indistinguishable.

Below, we define the $2 \mathrm{D}$ power spectrum of the turbulence $E\left(k_{\|}, k_{\perp}\right)$ in terms of the velocity $\mathbf{u}$ and magnetic field B as

$E\left(k_{\|}, k_{\perp}\right)=\sum_{\mathbf{k}^{\prime}}\left(\frac{1}{2} \rho_{0} \mathbf{u}_{\mathbf{k}^{\prime}}^{*} \cdot \mathbf{u}_{\mathbf{k}^{\prime}}+\frac{1}{8 \pi} \mathbf{B}_{\mathbf{k}^{\prime}}^{*} \cdot \mathbf{B}_{\mathbf{k}^{\prime}}\right)$,

where $\mathbf{u}_{k}=\mathcal{F}_{\mathbf{k}}\{\mathbf{u}\}$ and $\mathbf{B}_{k}=\mathcal{F}_{\mathbf{k}}\{\mathbf{B}\}$ are the $\mathbf{k}=\left(\mathbf{k}_{\|}+\mathbf{k}_{\perp}\right)$ components of the discrete Fourier transform of $\mathbf{u}$ and $\mathbf{B}$, respectively, $\mathbf{k}_{\|}=\left(\mathbf{k} \cdot \mathbf{B}_{0}\right) \mathbf{B}_{0} / B_{0}^{2}$, $\rho_{0}$ is the mean density, and $k_{\|, \perp}=\left|\mathbf{k}_{\|, \perp}\right|$. The superscript ${ }^{*}$ means the complex conjugate, and the sum extends for all the discrete modes, $\mathbf{k}^{\prime}$, with components in the interval $k_{\|} \leqslant\left|\mathbf{k}_{\|}^{\prime}\right|<\left(k_{\|}+1\right)$, and $k_{\perp} \leqslant\left|\mathbf{k}_{\perp}^{\prime}\right|<\left(k_{\perp}+1\right)$. The 1D power spectrum $E\left(k_{\perp}\right)$ is defined by

$E\left(k_{\perp}\right)=\sum_{k_{\|}=0}^{k_{\|, \max }} E\left(k_{\|}, k_{\perp}\right)$,

such that

$E_{\text {turb }}=\frac{1}{2} \rho_{0}\left\langle\mathbf{u}^{2}\right\rangle+\frac{1}{8 \pi}\left\langle\mathbf{B}^{2}\right\rangle=\sum_{k_{\perp}=1}^{k_{\perp, \max }} E\left(k_{\perp}\right)$,

is the total turbulent energy in the system, and the brackets, $\langle\cdot\rangle$, represent average in space.

The transfer spectrum is obtained from the following procedure: we multiply $\mathbf{u}_{\mathbf{k}}^{*}$ to the Fourier transform of the momentum equation (assuming the incompressible limit by ignoring the density variations), and we add the Fourier transform of the induction equation multiplied by $\mathbf{B}_{\mathrm{k}}^{*} /(4 \pi)$. We denote by $T_{\mathbf{k}}$ the time derivative of $\left(\rho_{0} \mathbf{u}_{\mathbf{k}}^{*} \cdot \mathbf{u}_{\mathbf{k}} / 2+\mathbf{B}_{\mathbf{k}}^{*}\right.$. $\left.\mathbf{B}_{\mathbf{k}} / 8 \pi\right)$, when we neglect the forcing and dissipation terms:

$$
\begin{aligned}
T_{\mathbf{k}}= & \Re\left\{\rho_{0} \mathbf{u}_{\mathbf{k}}^{*} \cdot \mathcal{F}_{\mathbf{k}}\left[(\mathbf{u} \cdot \nabla) \mathbf{u}-\frac{\mathbf{1}}{4 \pi}(\nabla \times \mathbf{B}) \times \mathbf{B}\right]\right\} \\
& -\frac{1}{4 \pi} \Re\left\{\mathbf{B}_{\mathbf{k}}^{*} \cdot \mathcal{F}_{\mathbf{k}}[\nabla \times(\mathbf{u} \times \mathbf{B})]\right\},
\end{aligned}
$$

with $\Re$ denoting the real part of.

The perpendicular transfer spectrum $T\left(k_{\perp}\right)$ is then defined by

$T\left(k_{\perp}\right)=\sum_{\mathbf{k}^{\prime}} T_{\mathbf{k}^{\prime}}$,

with the sum extending over all the modes $\mathbf{k}^{\prime}$, with the perpendicular components, $\mathbf{k}_{\perp}^{\prime}$, in the interval $0 \leqslant\left|\mathbf{k}_{\perp}^{\prime}\right|<$ $\left(k_{\perp}+1\right)$ (see, for example, Alexakis, et al. 2007; Alexakis 2011) . The turbulence energy transfer is given by the maximum value of $T\left(k_{\perp}\right)$,

$T_{\text {turb }}=\max \left\{T\left(k_{\perp}\right)\right\}$.

As we are going to see in Section 4.2, Figure 11 shows the normalized $2 \mathrm{D}$ power spectrum distribution $E\left(k_{\|}, k_{\perp}\right)$ for the same models presented in Figures 1 and 2, in the left and right column, respectively. By observing the distribution of modes with the highest values of energy (around 


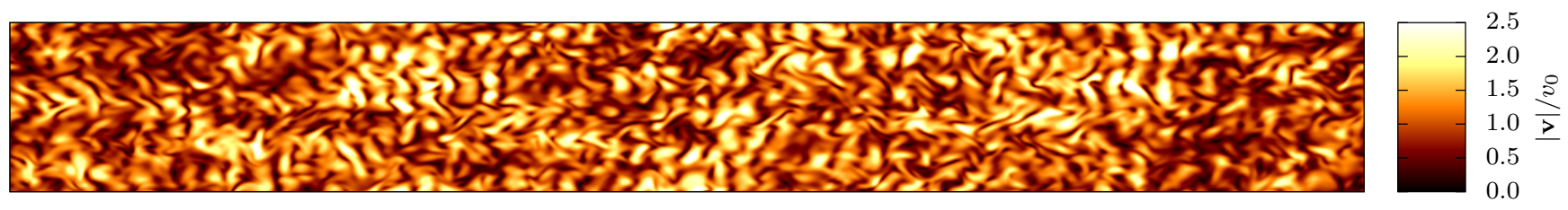

$8 \mathrm{~L}-\mathrm{Ms} 0.02-\mathrm{Ab}, M_{A}=0.4$

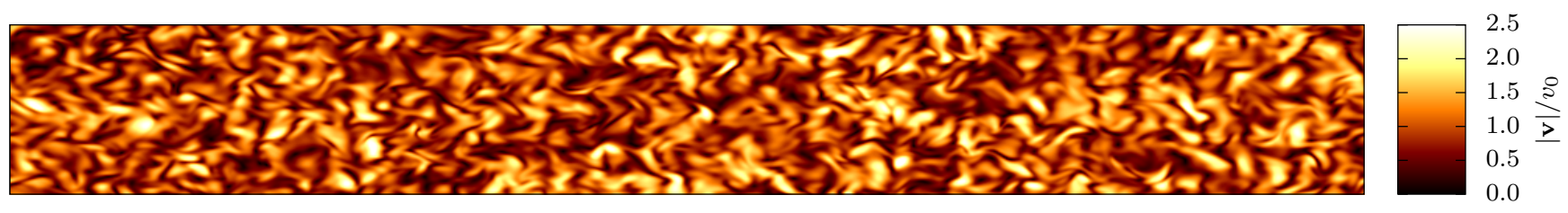

8L-Ms0.02-I, $M_{A}=0.4$

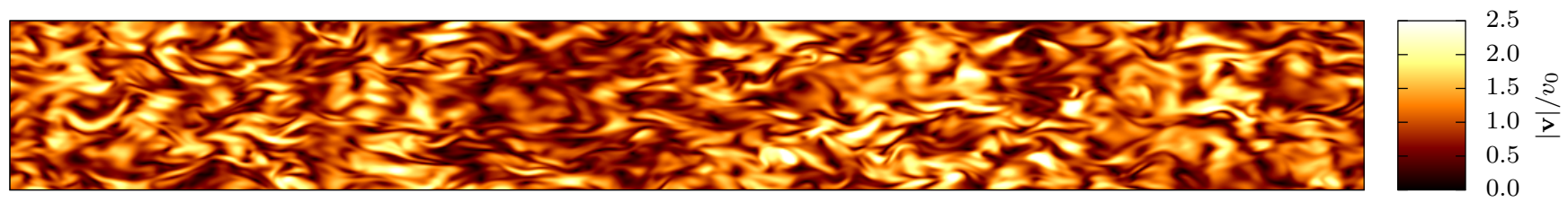

Figure 1. Central slice (xy-plane) showing the velocity modulus distribution at the final time of the simulations. Models with identical parameters but different forcing distributions in the k-space are compared. From top to bottom: A-forcing, Ab-forcing, and I-forcing. All simulations have the same Alfvénic Mach number $M_{A} \equiv v_{0} / v_{A, 0}=0.4$ and sonic Mach number $M_{S}=0.02$. See Table 1 for the complete description of the simulations parameters.

8L-Ms0.02-A, $M_{A}=0.2$

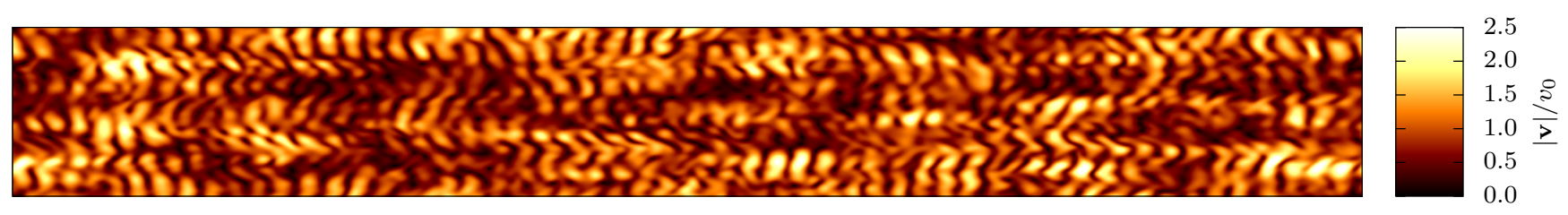

8L-Ms0.02-Ab, $M_{A}=0.2$

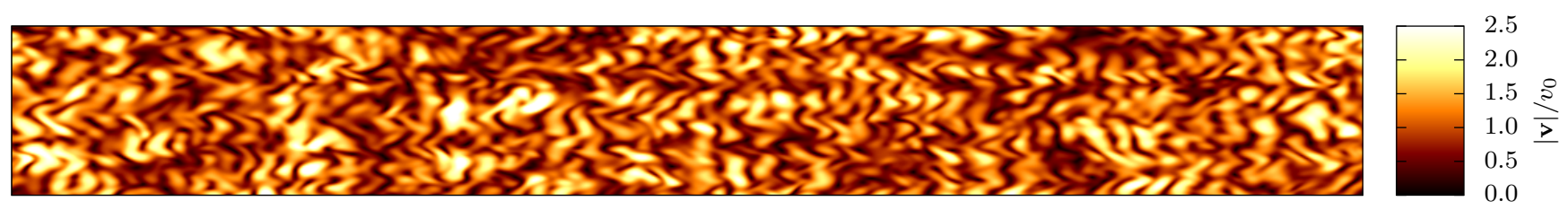

$8 \mathrm{~L}-\mathrm{Ms} 0.02-\mathrm{I}, M_{A}=0.2$

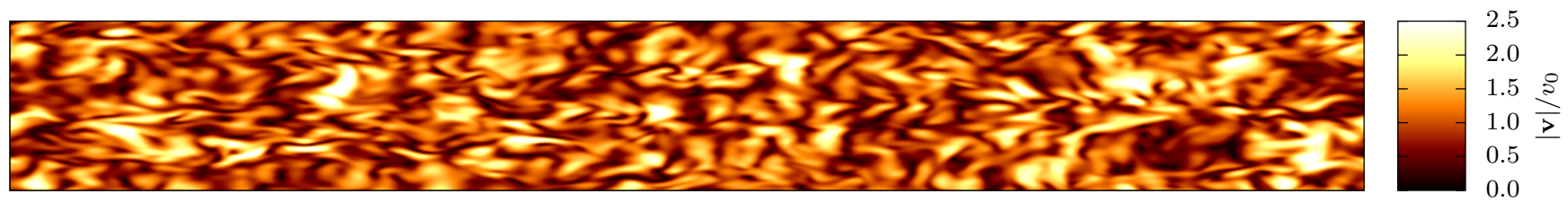

Figure 2. Same as Figure 1, but for simulations with the Alfvénic Mach number $M_{A} \equiv v_{0} / v_{A, 0}=0.2$. 


\section{Santos-Lima, Guerrero, de Gouveia Dal Pino $\&$ Lazarian}

$\left.0<k_{\|} L / 2 \pi, k_{\perp} L / 2 \pi<4\right)$, we can qualitatively assess the differences between the three different forcing schemes.

Observe that in the above definitions of energy and transfer spectrum, we are neglecting any density fluctuations. We choose to do so, in order to simplify the analysis and because we expect the Alfvén modes to dominate the turbulence spectrum for the subsonic simulations presented in this work.

\section{RESULTS}

\subsection{Compressibility and domain size effects}

Left panels of Figures 3 and 4 show the dependence of $\eta_{\mathrm{tf}}$, computed with the test-field method, with the $M_{A}$ resulting in the simulations (see for example Schrinner, et al. 2005; Brandenburg, Rädler \& Schrinner 2008). Each point in the curves corresponds to one model in Table 1 with anisotropic forcing (A), and points with the same shape and color correspond to models with the same sonic Mach number in Figure 3, and same box size in Figure 4. The values of the diffusivity are normalized by the estimate of the hydrodynamical turbulent diffusivity $\eta_{\text {hyd }}=(1 / 3) \ell v_{\text {rms }}$, where $\ell$ is the injection scale of the simulation (corresponding to the minimum wavenumber $k_{\perp} L / 2 \pi$ indicated in Table 1 ). The Alfvénic Mach number measured from the simulations is defined by, $M_{A}=v_{\mathrm{rms}} /\left\langle v_{A}\right\rangle$, where $\left\langle v_{A}\right\rangle=\left\langle B /(4 \pi \rho)^{1 / 2}\right\rangle$ is the Alfvén velocity averaged in the domain. The quantities $v_{\text {rms }},\left\langle v_{A}\right\rangle$, and $\eta_{\mathrm{tf}}$ represent time averaged values of the respective space averaged quantities.

The left panel of Figure 3 compares models with the same domain size $16 \mathrm{Lx} 1 \mathrm{~L}$, but three different values of sonic Mach number, $M_{S}=v_{0} / c_{s}$ (where $c_{s}$ is the isothermal sound speed of the simulation). For $M_{A}$ larger than a certain value $M_{A}^{*}\left(M_{A}^{*} \approx 0.5,0.4\right.$, and 0.2 for $M_{S}=0.32,0.08$, and 0.02 , respectively), we observe the approximate relation $\eta_{\text {tf }} / \eta_{\text {hyd }} \sim M_{A}^{3}$. For $M_{A}<M_{A}^{*}$, this power-law dependence with $M_{A}$ seems to change asymptotically to $\eta_{\mathrm{tf}} / \eta_{\mathrm{hyd}} \sim M_{A}^{2}$, with the constant of proportionality increasing with $M_{S}$. For the models with the lowest Mach number $\left(M_{S}=0.02\right)$ the value of $M_{A}^{*}$ is closer to the theoretical lower limit for validity of the classical weak turbulence (Equation 11). This limit is indicated by the vertical solid line in Figure 3.

The turbulent diffusion is naturally dominated by the motions of the injection scales, and the decorrelation time of the velocity fields at these scales can be expected to be directly related to the energy transfer time shown in the right panels of Figures 3 and 4 . If the break of the dependence $\eta_{\mathrm{tf}} / \eta_{\mathrm{hyd}} \sim M_{A}^{3}$ around some $M_{A}^{*}$ is caused by a change in the regime of the turbulence cascade (see Equation 6 ), the behavior of the energy transfer time $\tau_{\text {ener }}$ (i.e., the time-scale for the energy in the injection scale to cascade to smaller scales) could also occur around the same values of $M_{A}^{*}$. We estimated $\tau_{\text {ener }}$ dividing the total turbulence energy $E_{\text {turb }}=E_{v}+E_{b}$, where $E_{v}$ and $E_{b}$ are the kinetic and magnetic energies (in the incompressible limit), by the maximum value of the energy transfer spectrum, $T_{\text {turb }}$ (which is approximately the energy transfer rate at the injection scale or the turbulence injection power; see below the description of the energy transfer spectrum, $T\left(k_{\perp}\right)$, in the right columns of Figures 5 and 10). Right panel of Figure 3 shows the de- pendence of $\tau_{\text {ener }}$ (normalized by the estimate of the nonlinear turbulence time $\tau_{\mathrm{nl}}=\ell / v_{\mathrm{rms}}$ at the injection scale), as a function of $M_{A}$, for the same models shown in the left panel. In fact, $\tau_{\text {ener }} / \tau_{\text {nl }}$ follows approximately a well defined power law in $M_{A}$ for $M_{A}>M_{A}^{*}$, and becomes flatter for smaller values of $M_{A}$. The resulting power law does not coincide with that in the inertial range predicted for the weak turbulence regime, $\tau_{\text {ener }} / \tau_{\mathrm{nl}} \sim M_{A}^{-1}$. Instead, we obtain a dependency $\sim M_{A}^{-2}$.

Models with the same sonic Mach number, $M_{S}=0.02$, but different domain sizes are compared in the left panel of Figure 4 . The shorter the parallel extension $L_{\|}$of the domain, the larger the departure from the relation $\eta_{\mathrm{tf}} / \eta_{\text {hyd }} \sim$ $M_{A}^{3}$ for $M_{A}$ smaller than some $M_{A}^{*}$, which increases with the decrease of the domain size. The theoretical limits for the validity of the classical weak turbulence indicated by the vertical lines in Figure 3 are also shown in Figure 4. Each color corresponds to a different domain size (following the same color scheme for each set of simulations). We also observe in the right panel of Figure 4 that for simulations with $L_{\|}<16 \mathrm{~L}$, the curve for $\tau_{\text {ener }} / \tau_{\mathrm{nl}}$ deviates from a powerlaw for increasing values of $M_{A}^{*}$.

In the left panels of Figure 5 we present the $1 \mathrm{D}$ power spectrum of the total energy, $E\left(k_{\perp}\right)$, for simulations with different $M_{A}$. Inside these same panels we also show the ratio between the velocity and the magnetic field power spectra, $E_{\mathbf{u}}\left(k_{\perp}\right) / E_{\mathbf{B}}\left(k_{\perp}\right)$. On the right column we present the energy transfer spectrum, $T\left(k_{\perp}\right)$, for the same models, normalized by $E_{\mathrm{turb}} / \tau_{\mathrm{nl}}$, which is the expected value of the energy transfer spectrum in the case of strong turbulence cascade. The presence of a plato in the energy transfer spectrum is indicative of an inertial range, where the energy flux between scales is constant. The flatness of $T\left(k_{\perp}\right)$ just after the injection scale $k_{\perp} L / 2 \pi \approx 3-4$ is important to guarantee that with the current resolution, the energy transfer time from this scale (and therefore the velocity correlation time) is shorter than the dissipation time. If this were not the case, the measured $\eta_{t f}$ could be dominated by the numerical effective viscosity, as the velocity decorrelation time would be of the order of the dissipation time (see Equation 6). The top panels show simulations with the highest $M_{S}=0.32$, and domain size $16 \mathrm{Lx} 1 \mathrm{~L}$. The middle panels show models with the smallest $M_{S}=0.02$. In both sets, the power spectrum of the model with $M_{A}$ closer to unity shows a poor inertial range $\left(4 \lesssim k_{\perp} L / 2 \pi \lesssim 10\right.$ ). For this wavenumber interval, the normalized transfer spectrum decays roughly ten percent for the model with $M_{S}=0.02$ (middle panel). As $M_{A}$ decreases, a gradual steepening in the spectrum is accompanied by the decrease on the energy transfer values at the injection scale, which is consistent with the increase of the energy transfer time. The power law index seems to become steeper than -2 (the value predicted for the weak turbulence regime) for the models with $M_{S}=0.02$ (middle panels). The transfer spectra for the simulations with $M_{S}=0.02$ (middle panel) do not reveal a clear inertial range for the models with $M_{A}=0.2$ and $M_{A}=0.1$. Nonetheless, the microscopic (or numerical) dissipation does not seem to be predominant at the perpendicular wavenumbers just above the injection wavenumber $k_{\perp} L / 2 \pi \approx 4$. We see that the transfer spectra have not been reduced significantly until the wavenumbers above $k_{\perp} L / 2 \pi \approx 7$. The impact of the dissipation level close to the injection scale will be analyzed in Section 4.1 through 

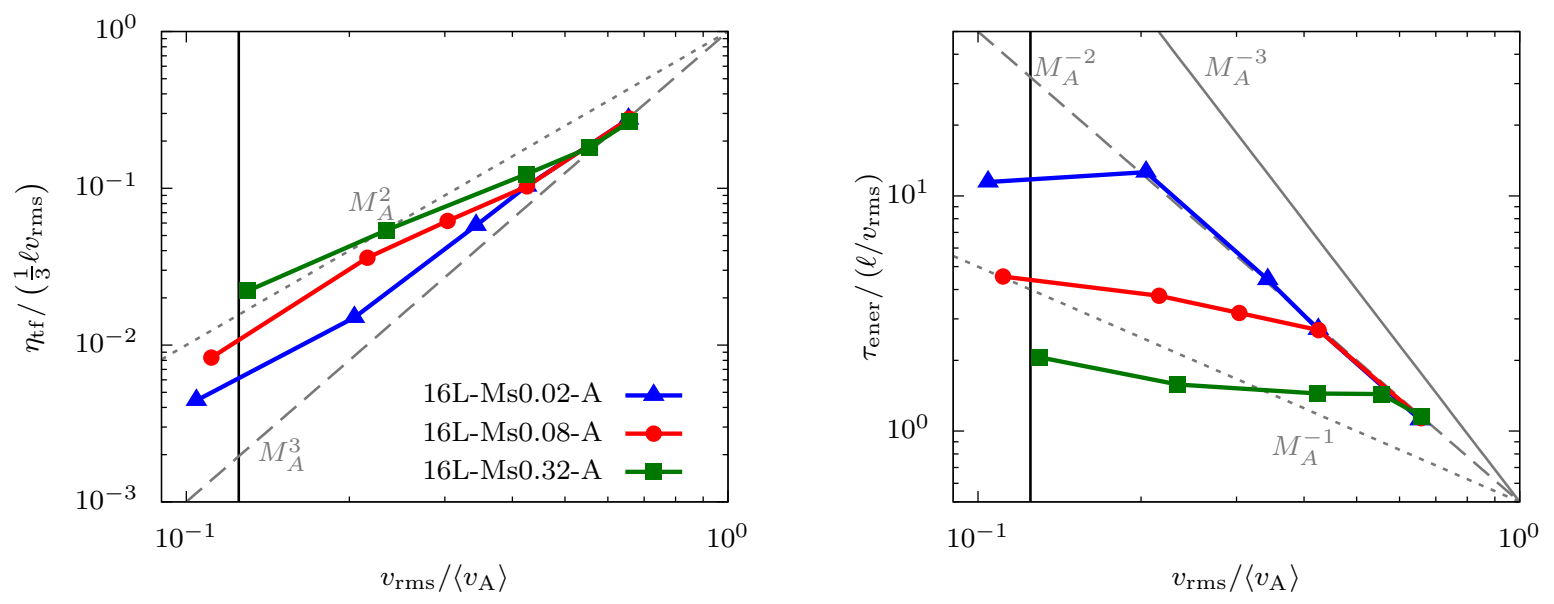

Figure 3. Magnetic diffusion coefficients $\eta_{\mathrm{tf}}$ measured by the test-field method (left) and the energy transfer time $\tau_{\text {ener }} \equiv E_{\text {turb }} / T_{\text {turb }}$ (right), as a function of the Alfvénic Mach number $M_{A}=v_{\mathrm{rms}} /\left\langle v_{A}\right\rangle$. Simulations with the same A-forcing, the domain sizes, but different sonic Mach number $M_{S}=v_{\mathrm{rms}} / c_{s}$ are compared. Each point corresponds to one run in Table 1. For the parameters used in these simulations the vertical solid line indicates the lower limit of $M_{A}$ given by Eq. 11 at the injection scale $\ell$.
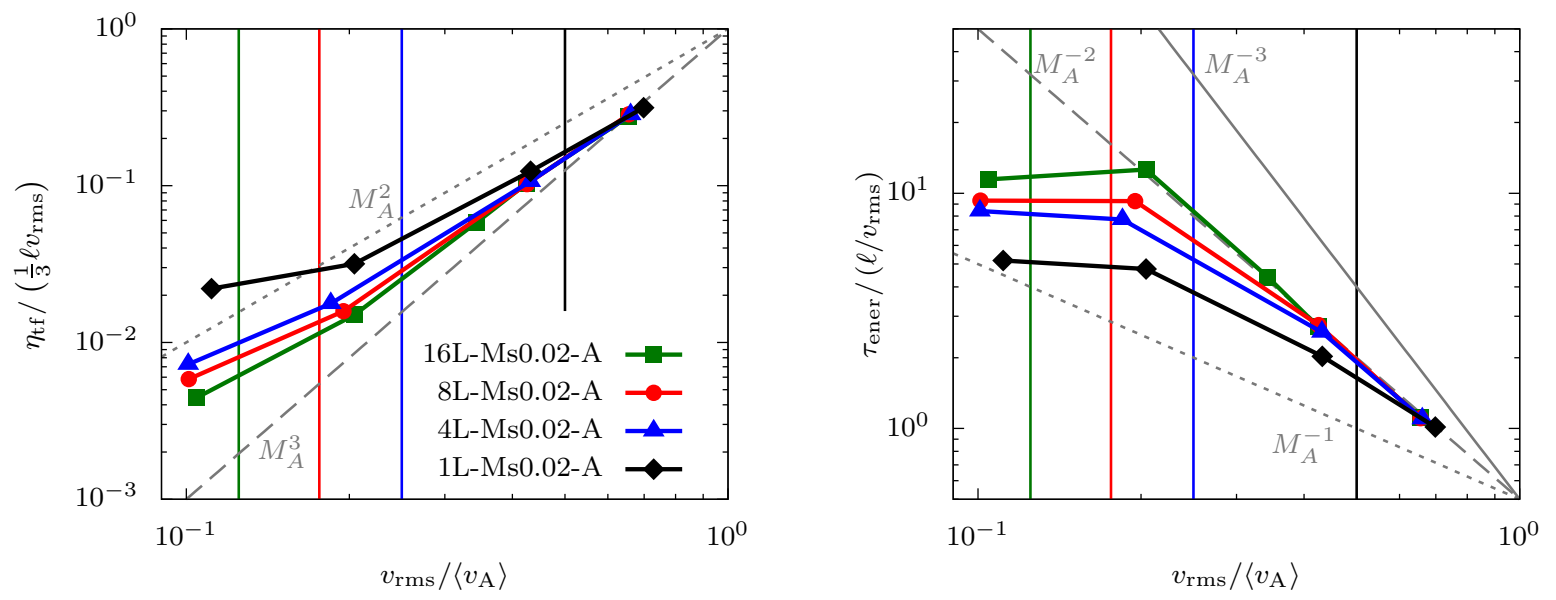

Figure 4. Magnetic diffusion coefficients $\eta_{\mathrm{tf}}$ measured by the test-field method (left) and the energy transfer time $\tau_{\text {ener }} \equiv E_{\text {turb }} / T_{\text {turb }}$ (right), as a function of the Alfvénic Mach number $M_{A}=v_{\mathrm{rms}} /\left\langle v_{A}\right\rangle$. Simulations with the same A-forcing, the same sonic Mach number $M_{S}=0.02$, but different domain sizes are compared. Each point corresponds to one run in Table 1. For each domain size (corresponding to different color curves), a vertical solid line indicates the lower limit of $M_{A}$ given by Eq. 11 at the injection scale $\ell$.

the comparison of models with different perpedicular resolutions. The bottom panels show models with $M_{S}=0.02$ and a domain size $1 \mathrm{Lx} 1 \mathrm{~L}$, for which the validity of the classical weak turbulence has the theoretical lowest limit around $M_{A}=0.5$. Below this value, the power spectrum does show the steepening seen in the model with extended domain (middle panel) with the decrease of $M_{A}$, and the transfer spectrum does not reduce substantially, which is consistent with a non increasing energy transfer time, confirming the previous analysis for these models (see the right panel of Figure 4).

The ratio between the kinetic and the magnetic power spectra shown in the left panels of Figure 5 is approxi- mately constant and close to unity, except for the largest wavenumbers, inside the dissipation range, where numerical effects dominate. When the energy spectrum is dominated by Alfvén modes, we should expect equipartition between the magnetic and kinetic energy spectra inside the inertial range, following the equality between these two amplitudes in each individual Alfvén mode. We observe this equipartition even for our models with larger compressibility (models 16L-Ms0.32-A; see top left panel in Figure 5).

Figure 6 compares the 2D energy spectrum for simulations from the same sets shown in Figure 5. On the left column the simulations have $M_{A}=0.4$, and on the right column $M_{A}=0.2$. Top and middle panels correspond to 
16L-Ms0.32-A

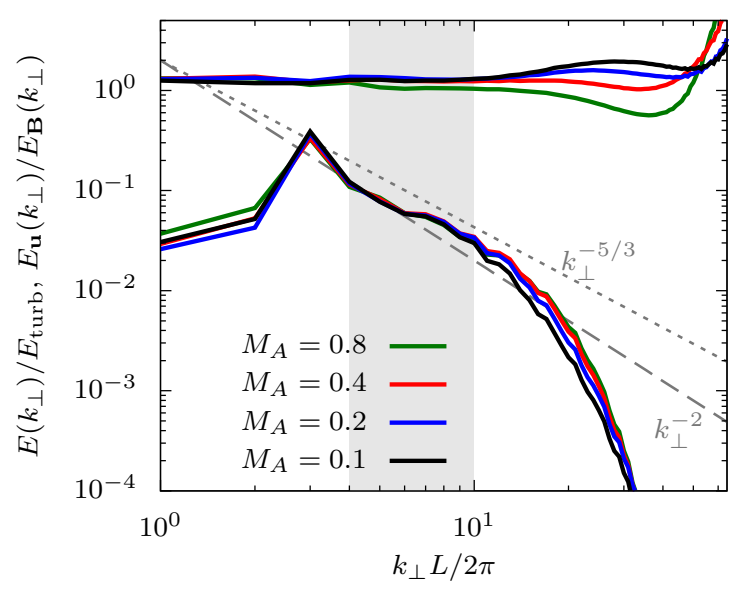

16L-Ms0.02-A

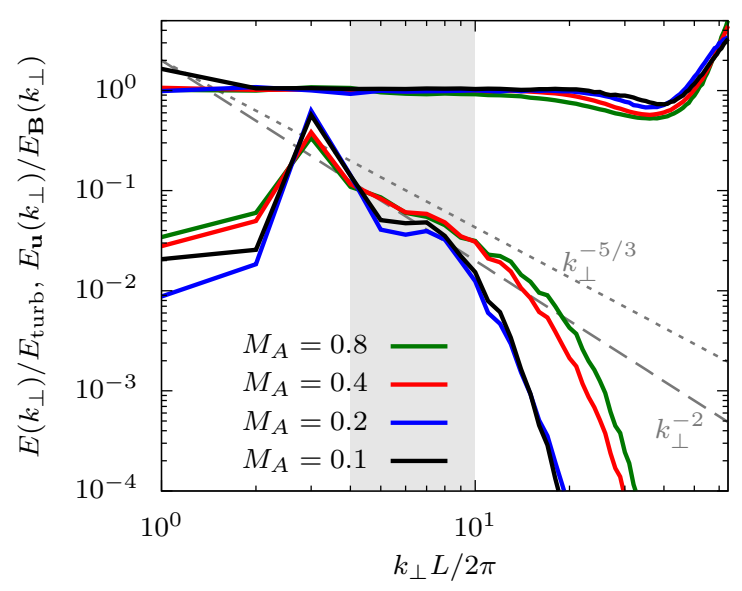

1L-Ms0.02-A

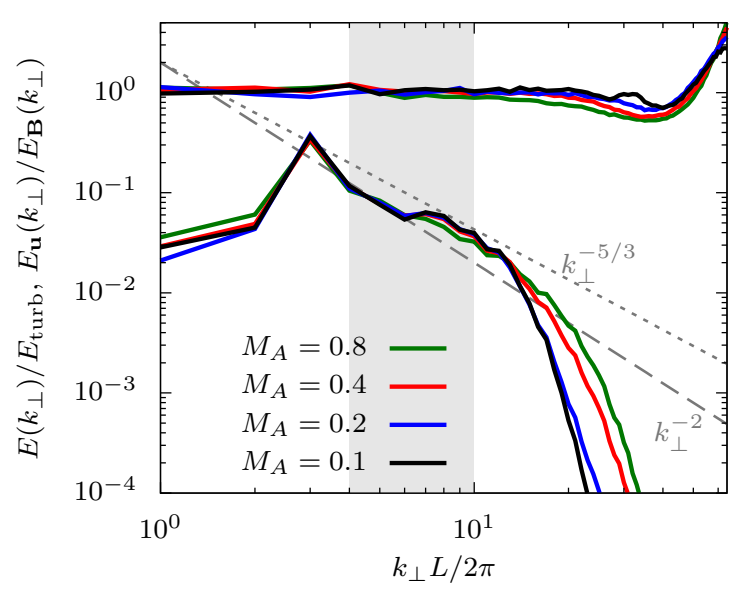

16L-Ms0.32-A

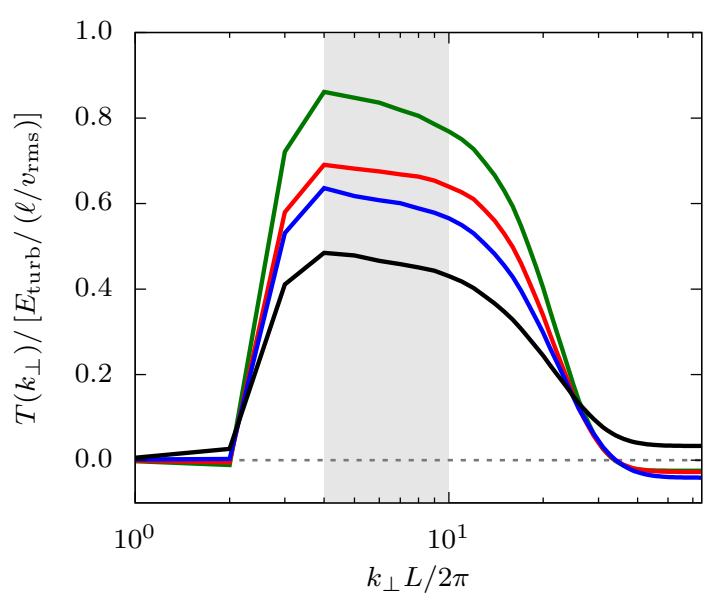

16L-Ms0.02-A

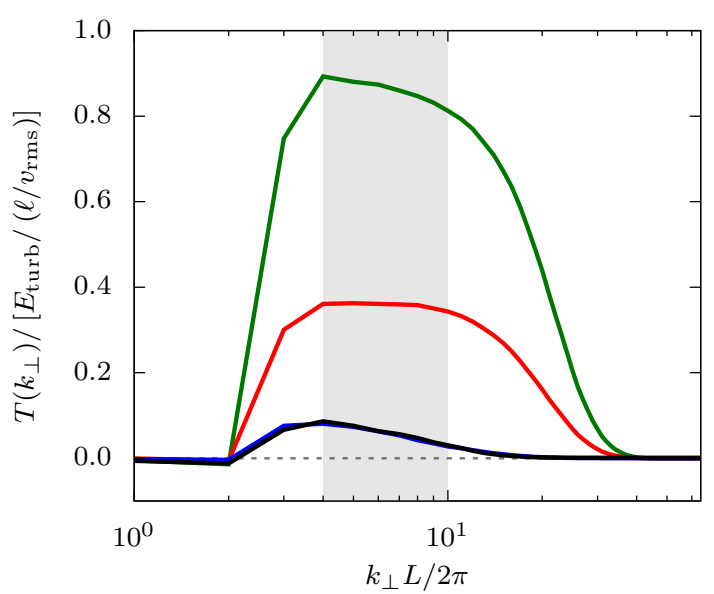

1L-Ms0.02-A

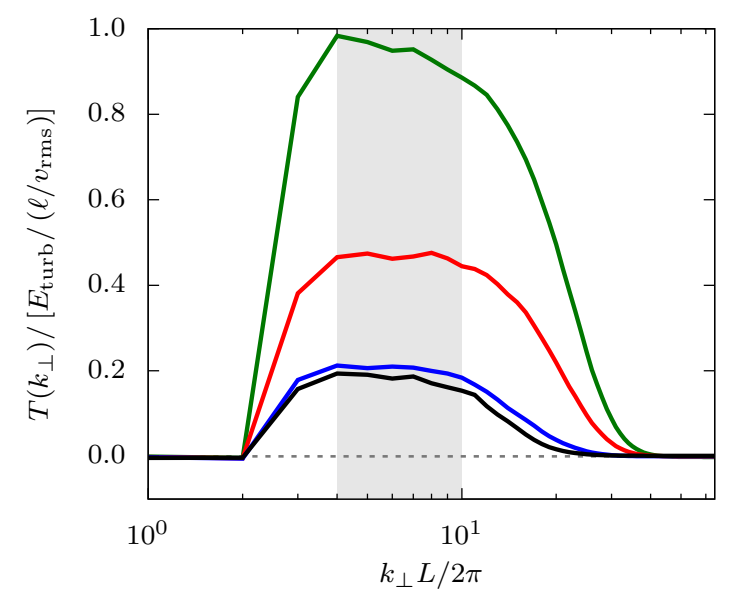

Figure 5. The energy spectrum $E\left(k_{\perp}\right)$ (left column) and the energy transfer spectrum $T\left(k_{\perp}\right)$ (right panel). The ratio between the velocity and the magnetic power spectra is also shown in the energy spectrum plots. In each panel, models from Table 1 with different Alfvénic Mach numbers $M_{A}$ are represented by curves with different colors. Top: models with sonic Mach number $M_{S}=0.32$ and domain size 16Lx1L; middle: models with $M_{S}=0.02$ and domain size $16 \mathrm{Lx} 1 \mathrm{~L}$; bottom: models with $M_{S}=0.02$ and domain size $1 \mathrm{Lx} 1 \mathrm{~L}$. The power laws $\propto k_{\perp}^{-5 / 3}$ and $\propto k^{-2}$ are also depicted for comparison in the left panels. The gray area covers the wavenumbers range $4<k_{\perp} L / 2 \pi<10$ for which the transfer spectrum is approximately constant and close to its maximum value. 
simulations with fixed domain size, $L_{\|}=16 \mathrm{~L}$, but different sonic Mach numbers, $M_{S}=0.32$ (top panels), and $M_{S}=0.02$ (middle panels). The less compressible simulations $\left(M_{S}=0.02\right)$ reveal two features which are not visible in the most compressible simulations $\left(M_{S}=0.32\right)$ : a suppression in the energy cascade in the parallel direction (vertical axis and steepening of the energy spectrum in the perpendicular direction (horizontal axis) when $M_{A}$ decreases. Both features are expected to emerge in the weak turbulence regime in the limit of incompressible MHD (Afvén waves turbulence). The bottom panels of Figure 6 correspond to simulations with the same compressibility as the middle panels $\left(M_{S}=0.02\right)$, but with a shorter domain size in the parallel direction $L_{\|}=1 \mathrm{~L}$. The two features described above for the simulations with larger domain size are clearly weaker in the simulations with shorter domain, which are out of the limit given by Equation 11 (at least near the injection scale).

We note in Figure 6 that the energy distributions in the parallel direction (vertical axis) present some peaks or "steps". This effect becomes stronger in the right panels, for which the mean magnetic field is stronger (smaller $M_{A}$ ). These peaks appear around the parallel wavenumbers which are harmonics of the wavenumber where the forcing amplitude is maximum, $k_{\|} L / 2 \pi=4$ (see Table 1 ). We attribute this feature to the peaked distribution of the forced modes on the parallel wavenumbers for the A-forcing scheme. At the same time, the wave-turbulence character becomes more pronounced when the mean magnetic field is stronger. Nonlinear coupling between triads of Alfvén waves naturally generates the higher order harmonics. The presence of these same harmonics in the energy spectrum was also pointed out in Ghosh et al. (2009), for the turbulence produced by a spectrum of Alfvén waves containing only one discrete parallel frequency (monochromatic) combined with quasi2D MHD modes (nearly zero Alfvén frequency) which has no broad enough spectrum around $k_{\|}=0$. In Figure 11 (discussed in the next subsection), it is shown that these steps are much less pronounced in the simulations with the Ab-forcing scheme, which has a broader and smoother distribution of amplitudes in the parallel wavenumbers. In this case, the non-linear coupling of waves involving a broader spectrum is enough to "fill" the gaps around the peaks seen before. Therefore, while the presence of the steps in the parallel spectrum of the simulations with the A-forcing makes the wave character of the turbulence visible, the resulting magnetic field diffusion and energy transfer time are not different from the models employing the Ab-forcing scheme, where the parallel spectrum is smooth.

Figure 7 shows, for the same set of simulations presented in the Figures 3 and 4, the dependence of the ratio between the amplitude of the $2 \mathrm{D}$ component of the solenoidal velocity field, $\left\langle v_{2 \mathrm{D}, \text { sol }}\right\rangle$ and the total $\mathrm{rms}$ velocity $v_{\mathrm{rms}}$, with $M_{A}$. We calculate $\mathbf{v}_{2}$, sol from the Fourier components of the velocity field, $\mathbf{v}_{\mathbf{k}}$, by removing the vector components parallel to both $\mathbf{k}$ and $\mathbf{B}_{0}$ (that is, the vector components which are either potential or parallel to the mean magnetic field), and finally keeping only the modes for which $k_{\|}=0$ ). The $2 \mathrm{D}$ velocity components are not excited by the A-forcing scheme, but they naturally develop in the system from the wave interactions. In the weak turbulence theory, the $2 \mathrm{D}$ modes are required as one component in the three-wave resonant interactions (although they can exist only in finite sized do- mains). As these velocities do not bend the magnetic field, they can easily mix the field lines in the perpendicular direction, similar to hydrodynamical motions $\left(\eta_{2 \mathrm{D}} \sim \ell v_{2 D}\right)$, dominating the diffusion rate. Similar to Figure 3 , the left panel of Figure 7 compares simulations with the same domain size 16Lx1L, but different compressibility $\left(M_{S}\right)$. Analogous to the behavior of $\eta_{\mathrm{tf}}$ for $M_{A}>M_{A}^{*}$, the values of $\left\langle v_{2 \mathrm{D} \text {,sol }}\right\rangle / v_{\mathrm{rms}}$ converge to an approximate power law in $M_{A}$, and deviate from this trend for $M_{A}<M_{A}^{*}\left(M_{A}^{*} \approx 0.5,0.4\right.$, and 0.2 for $M_{S}=0.32,0.08$, and 0.02 , respectively; we see that these values of $M_{A}^{*}$ correspond to the value of $M_{A}$ above which the energy transfer time follows the dependence $\tau \propto M_{A}^{-2}$, as seen in the right panel of Figure 3). Nonetheless, this analysis should be taken with caution because of the short range of values of the ratio $\left\langle v_{2 \mathrm{D}, \text { sol }}\right\rangle / v_{\text {rms }}$ and the statistical uncertainties generated by the fluctuations in the curves. Following the trend of $\eta_{\mathrm{tf}},\left\langle v_{2 \mathrm{D} \text {, sol }}\right\rangle / v_{\mathrm{rms}}$ also increases with $M_{S}$. The right panel of Figure 7 compares simulations with the same $M_{S}=0.02$, but different domain sizes. In this case all the sets of simulations show a similar qualitative behavior, i.e., $\left\langle v_{2 \mathrm{D}, \text { sol }}\right\rangle / v_{\text {rms }}$ increases with $M_{A}$. The magnitude of each curve is inversely proportional to the square root of $\mathrm{L}_{\|}$. In summary, the magnitude of $\left\langle v_{2 \mathrm{D} \text {,sol }}\right\rangle$ follows qualitatively that of $\eta_{\mathrm{tf}}$, but the dependence of the diffusivity with $M_{A}$ is stronger for $\eta_{\mathrm{tf}}$. There is no direct evidence that the mix caused by the $2 \mathrm{D}$ motions dominates the turbulent diffusivity, even for $M_{A}$ below $M_{A}^{*}$, for the simulations presented in Figures 3 and 4 .

In order to quantify the amount of turbulent energy in compressible modes for the simulations, for each wave vector in the Fourier space we performed the projection of the MHD variables onto the magnetosonic slow and fast eigenvectors. Figure 8 compares, for the same set of models presented in Figure 7, the ratio between the turbulent energy in each magnetosonic mode (slow and fast) and the total turbulent energy, as a function of $M_{A}$. The left panel compares models with different $M_{S}$ and fixed domain size 16Lx1L. For the most incompressible run set $\left(M_{S}=0.02\right)$, the relative energy in the slow modes is nearly constant with the different values of $M_{A}$, keeping close to 0.5 , and increases slightly with the decrease of $M_{A}$. The relative energy in the fast modes is about two orders of magnitude below that in the slow modes, and decreases slowly with the decrease of $M_{A}$. Only for the simulation with the smallest value of $M_{A}$ there is a sudden increase in the relative energy of the fast modes. The orther sets of simulations, with larger values of $M_{S}$, show a trend for the decrease in the relative energy of the slow modes with the decrease of $M_{A}$, at the same time that the relative energy of the fast modes increases. For the most compressible simulation $\left(M_{S}=0.32\right)$, for the smallest values of $M_{A}$ the energy in the fast modes overpass the energy of the slow modes. The right panel of Figure 8 compares models with fixed $M_{S}=0.02$ but different domain sizes. All sets of simulations behave similarly to the model with the largest domain size shown in the left panel.

\subsection{Forcing effects}

All the results described so far were derived from simulations using the turbulence forcing anisotropically distributed in the Fourier space (A-forcing). In order to test the sensitivity of these results to the forcing scheme, we have repeated 


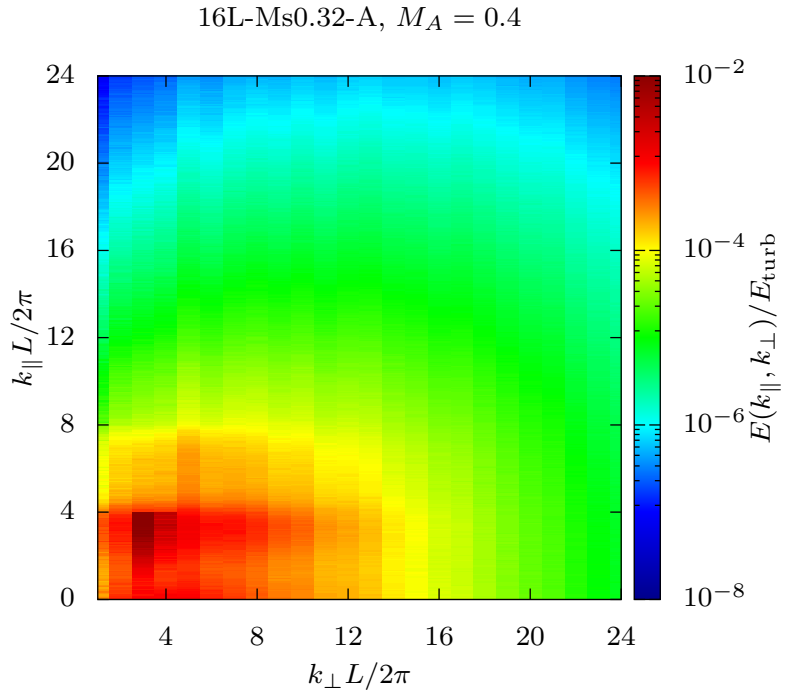

16L-Ms0.02-A, $M_{A}=0.4$

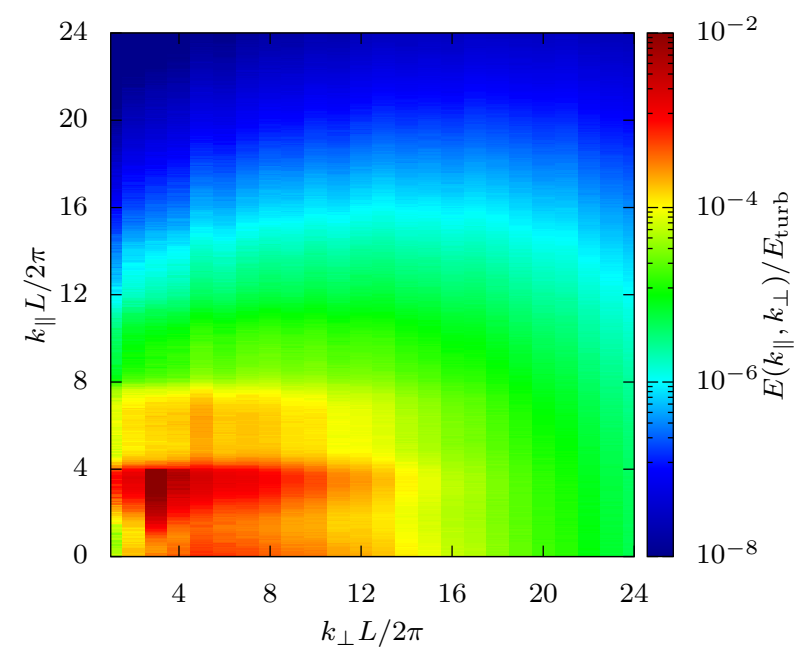

1L-Ms0.02-A, $M_{A}=0.4$

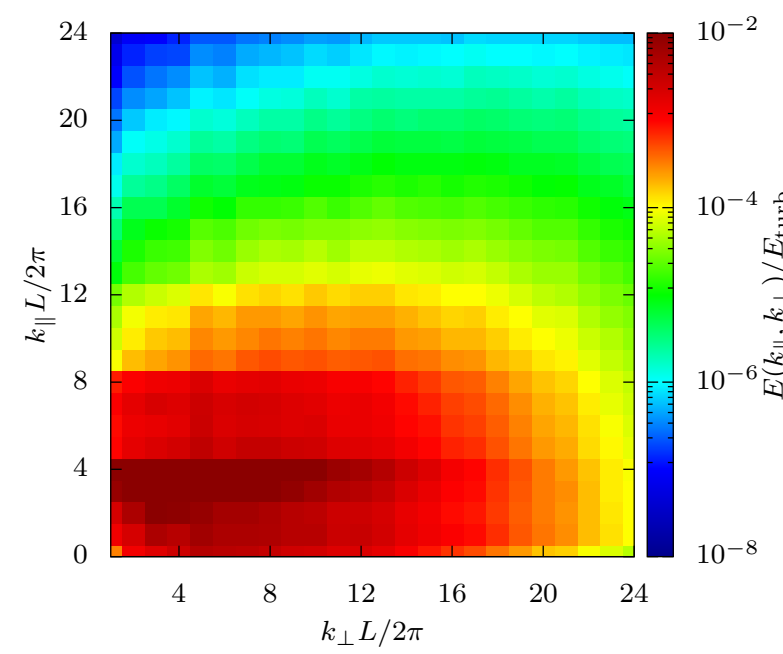

16L-Ms0.32-A, $M_{A}=0.2$

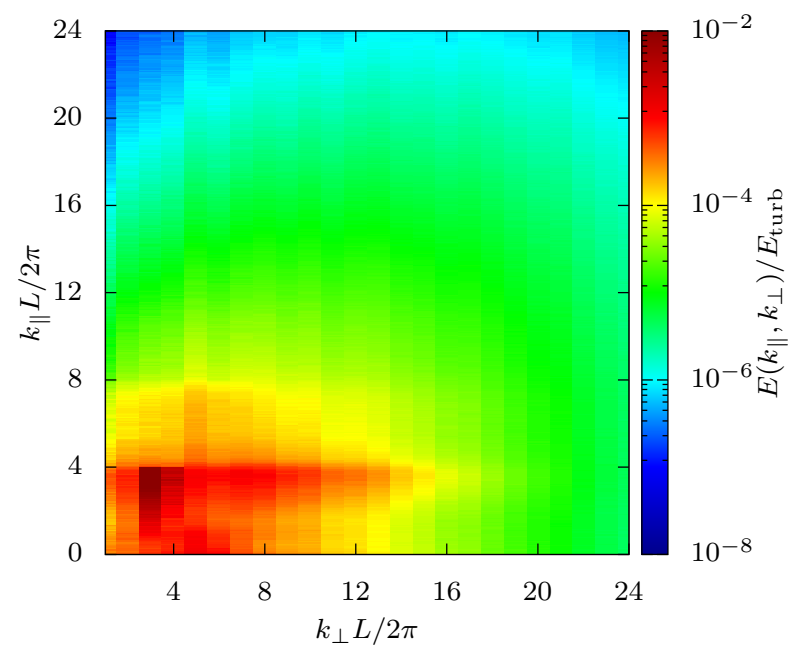

$16 \mathrm{~L}-\mathrm{Ms} 0.02-\mathrm{A}, M_{A}=0.2$

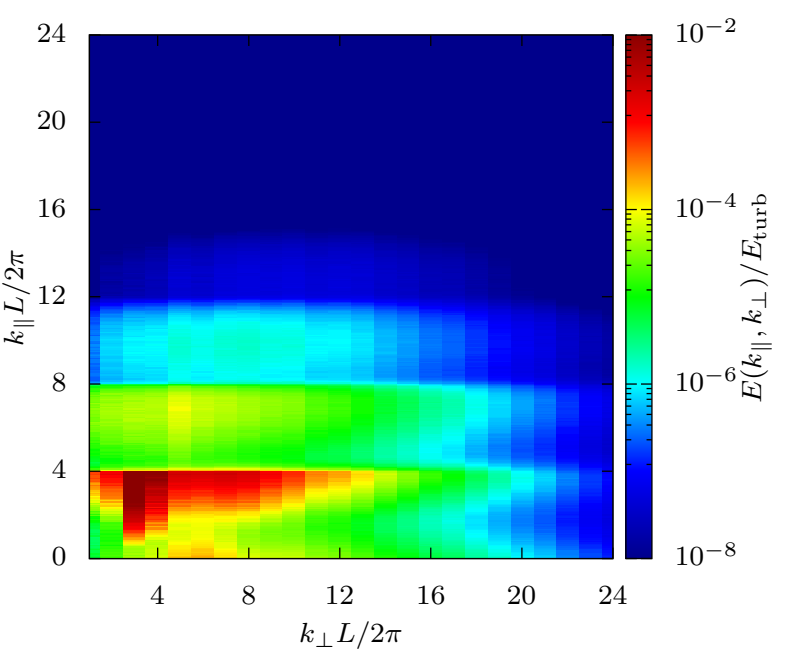

1L-Ms0.02-A, $M_{A}=0.2$

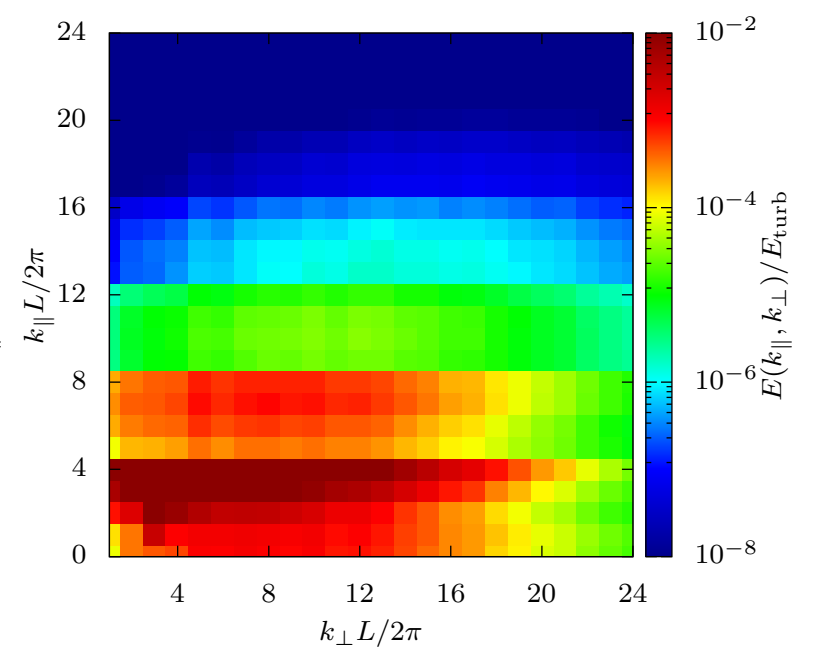

Figure 6. The $2 \mathrm{D}$ energy spectrum $E\left(k_{\|}, k_{\perp}\right)$ distribution in the $\left(k_{\|}, k_{\perp}\right)$ plane. Each column corresponds to a different nominal Alfvénic Mach number $M_{A, 0} \equiv v_{0} / v_{A, 0}$. Left column: $M_{A, 0}=0.4$. Right column: $M_{A, 0}=0.2$. Top row: domain size $L_{\|}=16 \mathrm{~L}$ and sonic Mach number $M_{S}=0.32$. Middle row: domain size $L_{\|}=16 \mathrm{~L}$ and sonic Mach number $M_{S}=0.02$. Bottom row: domain size $L_{\|}=1 \mathrm{~L}$ and sonic Mach number $M_{S}=0.02$. See Table 1 for the complete description of the simulations parameters. 

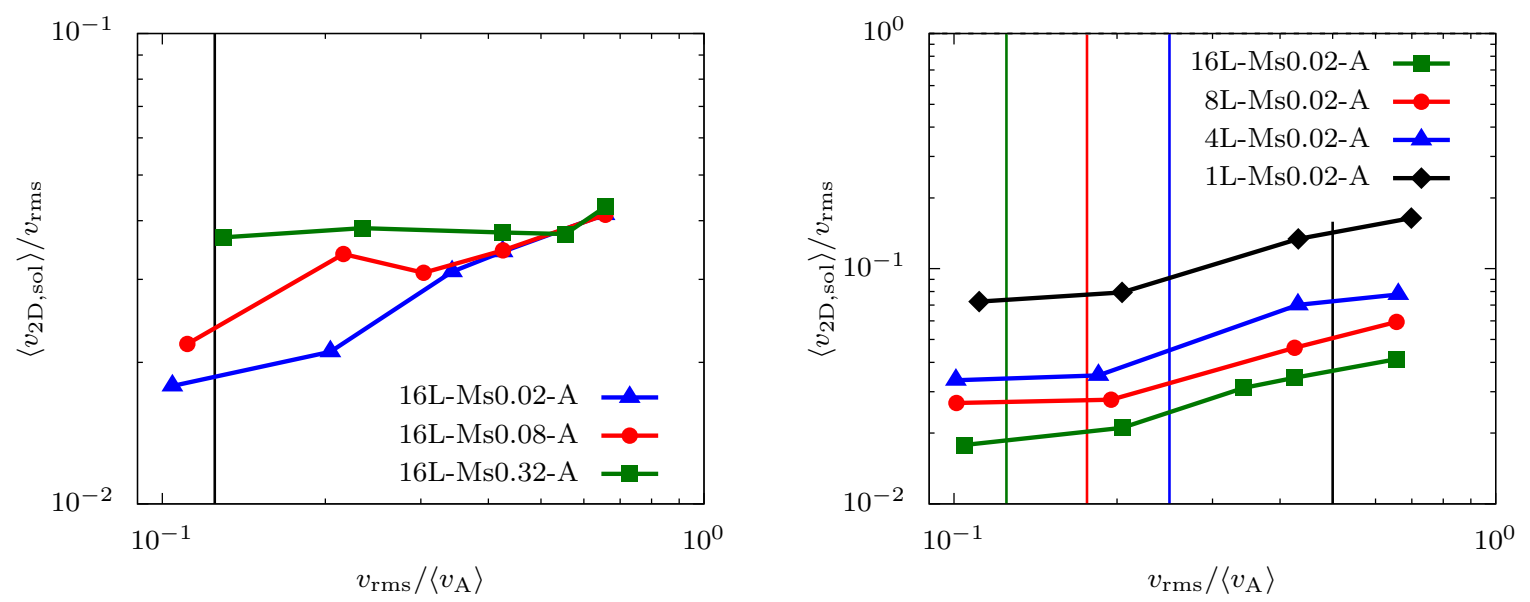

Figure 7. Ratio between the rms value of the $2 \mathrm{D}$ component of the solenoidal velocity $\left\langle v_{2 \mathrm{D}}\right.$,sol $\rangle$ and the total rms velocity $v_{\text {rms }}$, as a function of the Alfvénic Mach number $M_{A}=v_{\mathrm{rms}} /\left\langle v_{A}\right\rangle$. Left: simulations with different sonic Mach numbers $M_{S}=v_{0} / c_{s}$ are compared. Right: simulations with the same $M_{S}=0.02$, but different domain sizes are compared. Each point in the curves corresponds to a model in Table 1. For each domain size (corresponding to different color curves on the right panel), a vertical solid line indicates the lower limit of $M_{A}$ given by Eq. 11 at the injection scale $\ell$.
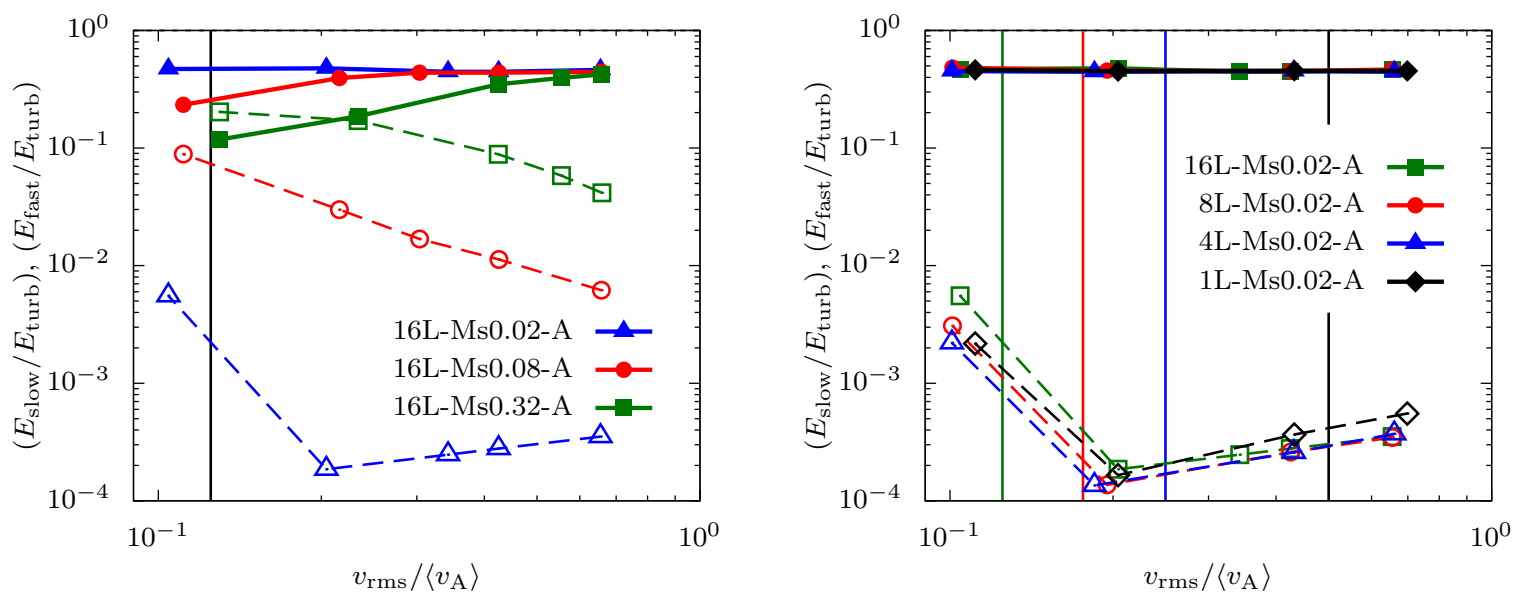

Figure 8. Ratio between the energy in each magnetosonic component of the turbulence and the total turbulent energy $E_{\text {turb }}$ as a function of the Alfvénic Mach number $M_{A}=v_{\mathrm{rms}} /\left\langle v_{A}\right\rangle$. The continuous lines are for the energy in the slow modes $E_{\text {slow }}$, and the dashed lines are for the energy in the fast modes $E_{\text {fast }}$. Left: simulations with different sonic Mach numbers $M_{S}=v_{0} / c_{s}$ are compared. Right: simulations with the same $M_{S}=0.02$, but different domain sizes are compared. Each point corresponds to a model in Table 1 . For each domain size (corresponding to different color curves on the right panel), a vertical solid line indicates the lower limit of $M_{A}$ given by Eq. 11 at the injection scale $\ell$.

the simulations with $M_{S}=0.02$ and domain size $8 \mathrm{Lx} 1 \mathrm{~L}$ using two alternative forcings, one isotropically distributed inside a spherical shell in the $\mathbf{k}$-space (I-forcing), and another where the amplitude of the modes inside a spherical shell are concentrated around $k_{\|} / k_{\perp}=1$ using the modulation factor $\propto\left(k_{\|} k_{\perp} / k^{2}\right)$ (Ab-forcing). This last forcing scheme can be thought as intermediate between the extreme A- and I-forcing cases (such as described in Section 3.1).

The left panel of Figure 9 shows that for simulations with the I-forcing, $\eta_{\mathrm{tf}}$ is essentially independent of $M_{A}$ for
$M_{A}<M_{A}^{*}=0.2$. Above $M_{A}^{*}$, this dependence follows a power law, $\eta_{\mathrm{tf}} / \eta_{\text {hyd }} \sim M_{A}^{-1}$, which is much weaker than the observed for the A-models, where the power low dependence is $\sim M_{A}^{-3}$. The Ab-models behave similar to the A-models, with scaling of $\eta_{\mathrm{tf}} / \eta_{\text {hyd }}$ between $\sim M_{A}^{-3}$ and $\sim M_{A}^{-2}$. In the right panel of Figure 9 we see that the energy cascading times, $\tau_{\text {ener }}$, around the injection scale, $\ell$, for the Ab-models are almost identical to those of A-models, at least for $M_{A}>$ $M_{A}^{*}$. In contrast, the increase of $\tau_{\text {ener }}$ with the decrease of 

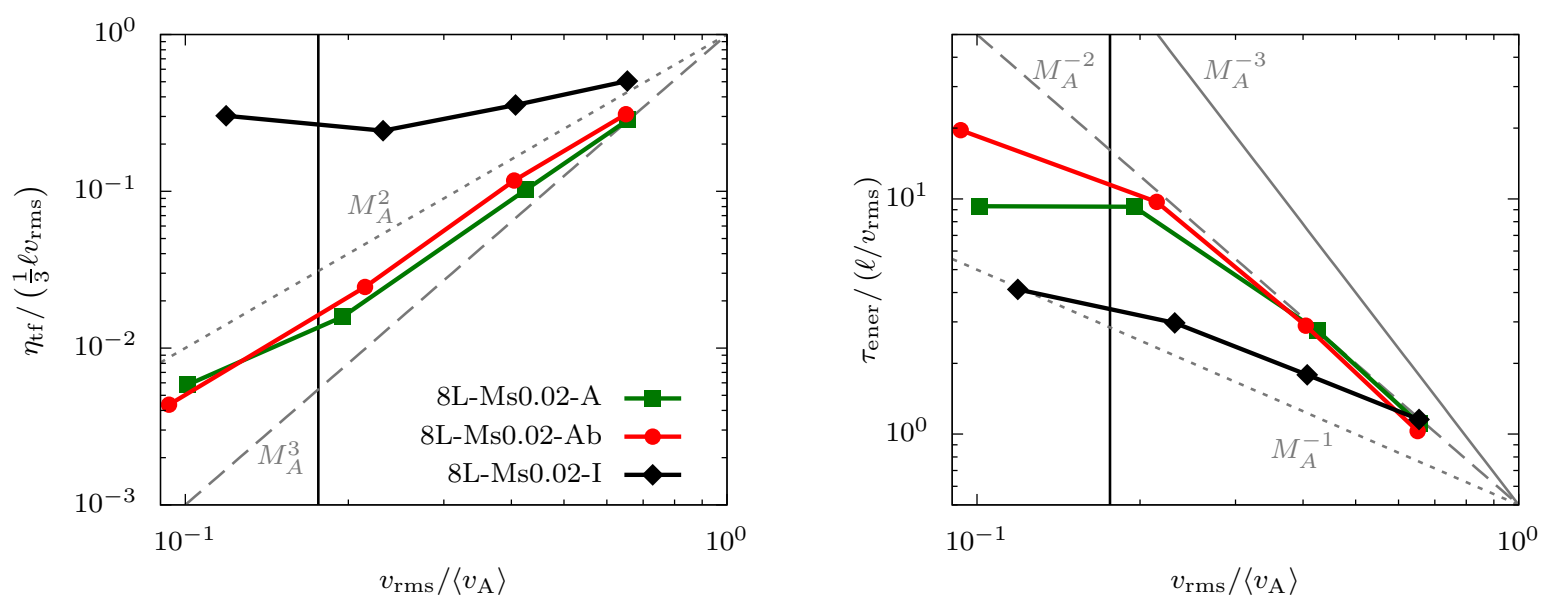

Figure 9. Magnetic diffusion coefficients $\eta_{\mathrm{tf}}$ measured by the test-field method (left) and the energy transfer time $\tau_{\text {ener }} \equiv E_{\text {turb }} / T_{\text {turb }}$ (right), as a function of the Alfvénic Mach number $M_{A}=v_{\mathrm{rms}} /\left\langle v_{A}\right\rangle$. Simulations with different turbulence forcing schemes are compared. All the runs have the same sonic Mach number $M_{S}=0.02$ and domain size $8 \mathrm{Lx} 1 \mathrm{~L}$. Each point corresponds to a model in Table 1 . For the parameters used in these simulations the vertical solid line indicates the lower limit of $M_{A}$ given by Eq. 11 at the injection scale $\ell$.

$M_{A}$ for the I-models is much slower than that for the Amodels.

The turbulence energy spectrum $E\left(k_{\perp}\right)$ for the simulations with I- and Ab-forcing are shown in the left column of Figure 10. Their respective energy transfer spectrum, $T\left(k_{\perp}\right)$, are shown in the right column. We do not notice the steepening of the energy spectrum with the decrease of $M_{A}$ for the I-models as seen in the Ab-models and A-models (Figure 5). Considering the analysis of both, the energy cascading time (right panel of Figure 9) and the energy transfer spectrum (right panel of Figure 10), there is a difference between the I-models and the $\mathbf{k}$-anisotropic forcing models $\mathrm{A}$ and $\mathrm{Ab}$. Notice that all the forcing schemes produce similar energy spectrum for $M_{A}=0.8$, therefore, the difference is manifested only in the presence of strong magnetic fields.

The 2D energy spectrum, $E\left(k_{\|}, k_{\perp}\right)$ for simulations with the different forcing schemes are compared in Figure 11. The top, middle and bottom panels correspond to the I, Ab, and A forcings, respectively. Simulations in the left column have $M_{A}=0.4$, and in the right column $M_{A}=0.2$. We note that substantial differences between models with different forcing become evident for smaller values of $M_{A}$. The $2 \mathrm{D}$ energy spectrum for values of $M_{A}$ closer to unity are almost indistinguishable (not shown). The k-anisotropic models (middle and bottom panels) show steepening in the energy distribution in the perpendicular direction (horizontal axis), while this effect is not observed in the simulations with I forcing (top panels). In all the cases we observe a sharp steepening of the spectrum in the parallel direction (vertical axis) for $M_{A}=0.2$.

With exception of the I-cases, the dependence of the diffusivity $\eta_{\mathrm{tf}}$ with $M_{A}$ for all the simulations with different forcings seems to be closely related to the behavior of $\tau_{\text {ener }}$ with $M_{A}$. Indeed, the left panel of Figure 12 shows that the values of $\left\langle v_{2 \mathrm{D} \text {,sol }}\right\rangle / v_{\mathrm{rms}}$ for the I-models are much larger compared to the other forcing schemes. There is no surprise in this fact, as the $2 \mathrm{D}$ velocity modes are only forced in the
I-models. These high $2 \mathrm{D}$ velocities can explain the diffusivity observed in the I-models, as $\eta_{\mathrm{tf}} / \eta_{\text {hyd }} \sim\left\langle v_{2 D, s o l} / v_{\mathrm{rms}}\right\rangle$.

Finally, the right panel of Figure 12 compares the relative turbulent energies in the magnetosonic slow and fast modes for the sets of simulations with the several forcings. No appreciable difference is observed between them.

\subsection{Resolution effects and convergence}

Figure 13 compares the model 16L-Ms0.02-A having resolution $2048 \times 128^{2}$ with the models $16 \mathrm{~L}-\mathrm{Ms} 0.02$-low-A and 16L-Ms0.02-hi-A, with identical parameters but resolutions $1024 \times 64^{2}$ and $2048 \times 256^{2}$, respectively. In order to make the higher resolution run feasible with the computational power we have available, the models 16L-Ms0.02-hi-A have twice the resolution only in the perpendicular direction to the uniform magnetic field. In the regime of weak turbulence, we expect (based on the theory) the non-linear energy transfer (turbulence cascade) to be more important in this direction, at least close to the injection scale.

Moreover, in Figure 13, we compare the low resolution models 16L-Ms-0.02-low-A with other two models, 16Lx2LMs-0.02-low-A and 16L-Ms0.02-low-A-diff2. The first of these models has double domain size in the direction perpendicular to the mean magnetic field $L_{\perp}$, that is, it has the ratio $L_{\perp} / \ell_{\perp}$ increased by a factor of two compared to the models 16L-Ms0.02-low-A. The other models, 16-Ms-0.02-lowA-diff2, have the hyper-viscosity and hyper-diffusivity replaced by the standard viscosity and resistivity, respectively (see the description of these microscopic diffusive terms in Sec. 3).

The minimum $M_{A}$ below which the 3D MHD turbulence becomes "enslaved" to the $2 \mathrm{D}$ modes is theoretically predicted to depend on the ratio $L_{\perp} / \ell_{\perp}$ (Nazarenko 2007; see Eq. 12). This minimum $M_{A}$ is indeed below the value delimiting the validity of the weak turbulence theory, which we have marked in our plots. Therefore, based on theory only, 
8L-Ms0.02-I

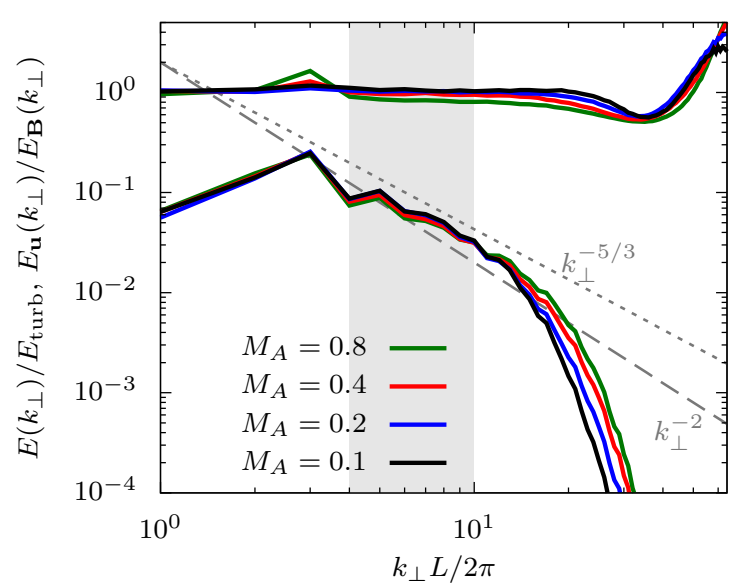

8L-Ms0.02-Ab

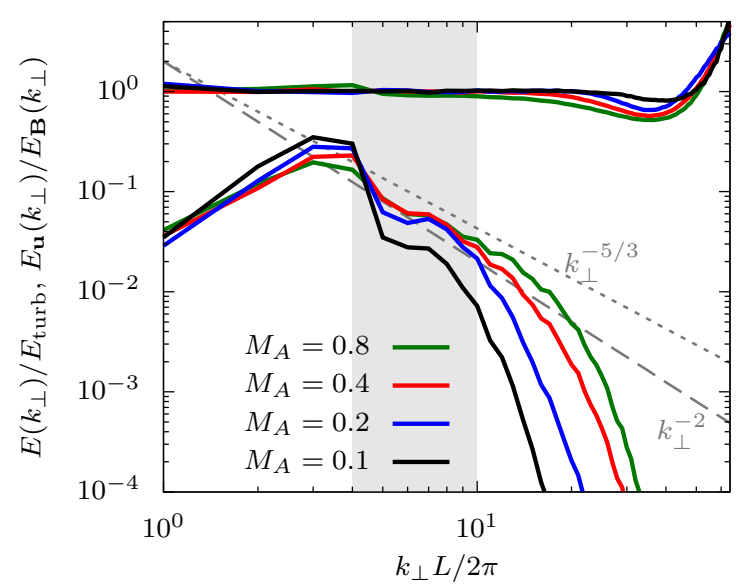

$8 \mathrm{~L}-\mathrm{Ms} 0.02-\mathrm{I}$

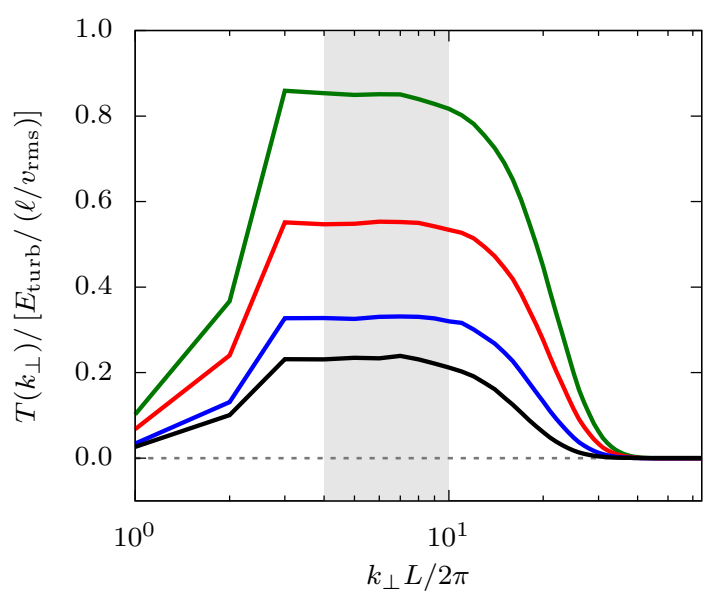

8L-Ms0.02-Ab

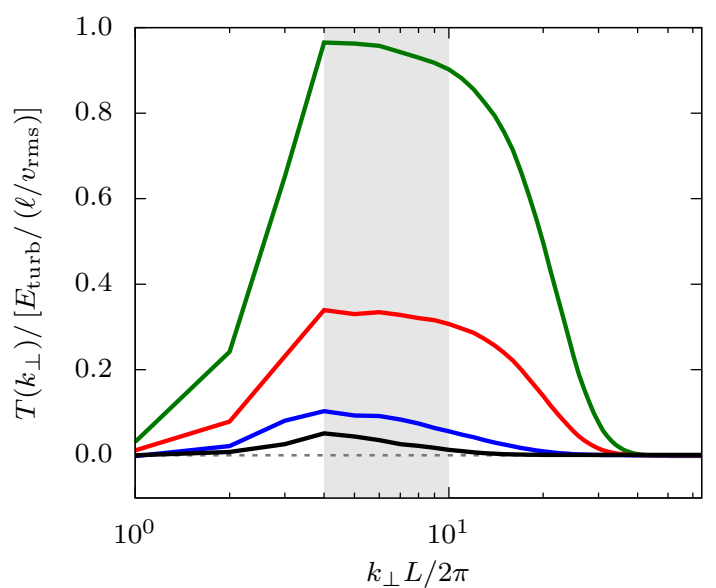

Figure 10. The energy spectrum $E\left(k_{\perp}\right)$ (left column) and the energy transfer spectrum $T\left(k_{\perp}\right)$ (right panel) for models with different turbulence forcing schemes. The ratio between the velocity and the magnetic field power spectra for each model is shown inside the energy spectrum plots. In each panel, models from Table 1 with different Alfvénic Mach numbers $M_{A}$ are represented by curves with different colors. All the runs have the same sonic Mach number $M_{S}=0.02$ and domain size $8 \mathrm{Lx} 1 \mathrm{~L}$. Top: isotropic forcing scheme $I$; bottom: anisotropic forcing scheme $A b$. The power laws $\propto k_{\perp}^{-5 / 3}$ and $\propto k^{-2}$ are also depicted for comparison in the left panels. The gray area covers the wavenumbers range $4<k_{\perp} L / 2 \pi<10$ for which the transfer spectrum is approximately constant and close to its maximum value.

we should not expect differences in the turbulence regime between the models 16L-Ms-0.02-low-A and 16Lx2L-Ms-0.02low-A. Nonetheless, because these theoretical expressions give only order of magnitude estimates, we present this convergence test for the perpendicular size of the domain, in order to check whether or not the length used in all the previous analyses influences our results.

The transport rate of large scale fields via reconnection diffusion (RD) is expected to be dominated by the statistics of the larger scale motions of the turbulence. Since the Reynolds and the magnetic Reynolds numbers of the turbulence flow are much larger than unity, the microphysics describing the diffusion mechanism (as for example ambipolar diffusion, ohmic resistivity, anomalous resistivity, or even "numerical diffusivity") should not have impact on the magnetic field diffusion coefficient (see Eq. 6). However, if the dif- fusion mechanism can somehow change the statistics of the turbulence close to the injection scale, then it can influence the RD process. The comparison between models 16L-Ms0.02-low-A and 16L-Ms0.02-low-A-diff2 aims to check if the hyper-viscosity and hyper-resistivity, used in all the other models in this work, could have some effect on the magnetic diffusivity coefficients.

The upper-left panel Fig. 13 shows the diffusion coefficients $\eta_{\mathrm{tf}}$ as a function of $M_{A}$ (see also left panel of Figure 3 ). The scaling laws are similar for the different resolutions. The change in the domains size $L_{\perp}$ also does not results in important changes in $\eta_{\mathrm{tf}}$. Finally, the results of the models using standard viscosity and hyperdifusivity (16L-Ms0.02-low-Adiff2) show no noticieable differences.

The low resolution models 16L-Ms-0.02-low-A contain runs with values of $M_{A}$ below the line indicating the lower 
8L-Ms0.02-I, $M_{A}=0.4$

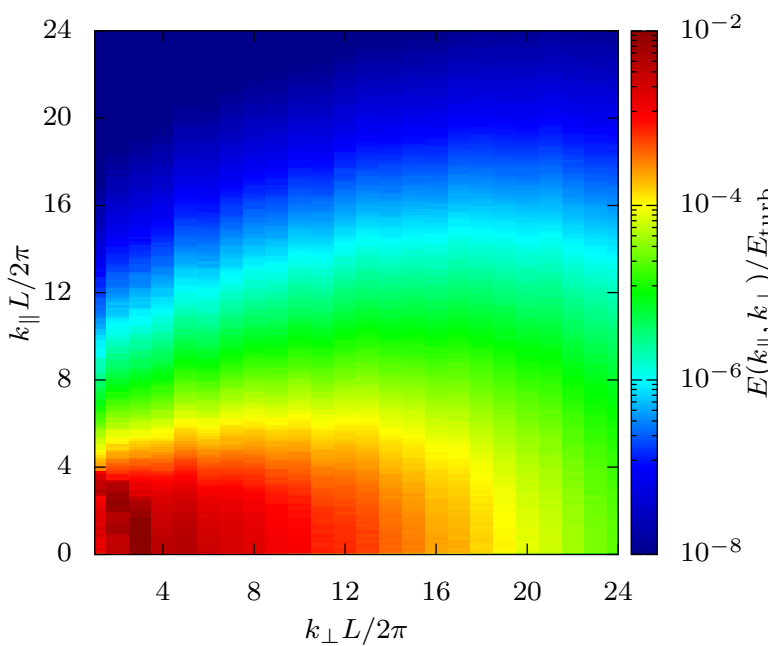

8L-Ms0.02-Ab, $M_{A}=0.4$

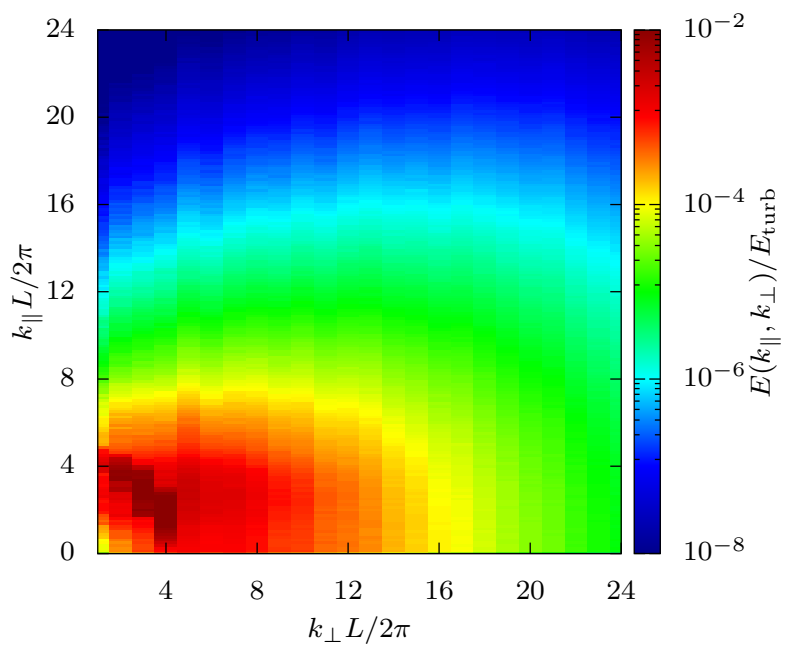

8L-Ms0.02-A, $M_{A}=0.4$

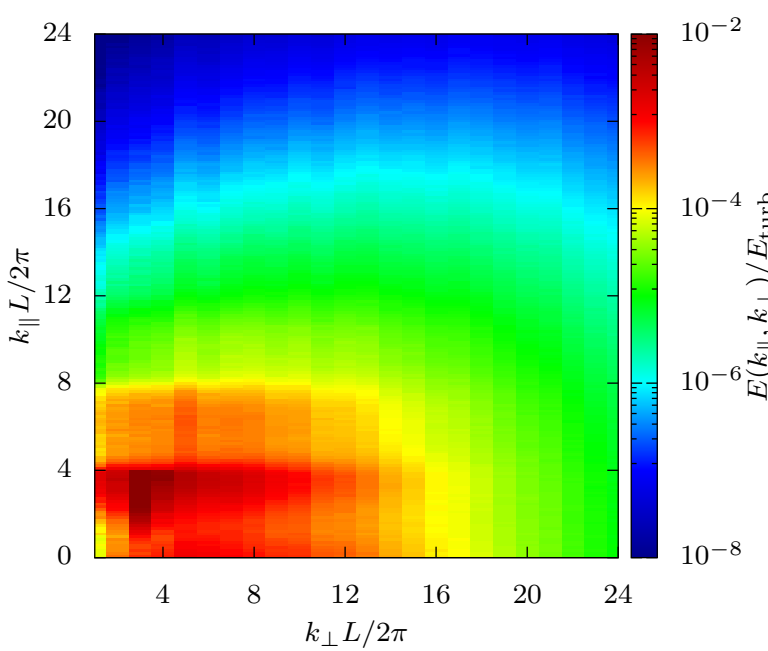

8L-Ms0.02-I, $M_{A}=0.2$

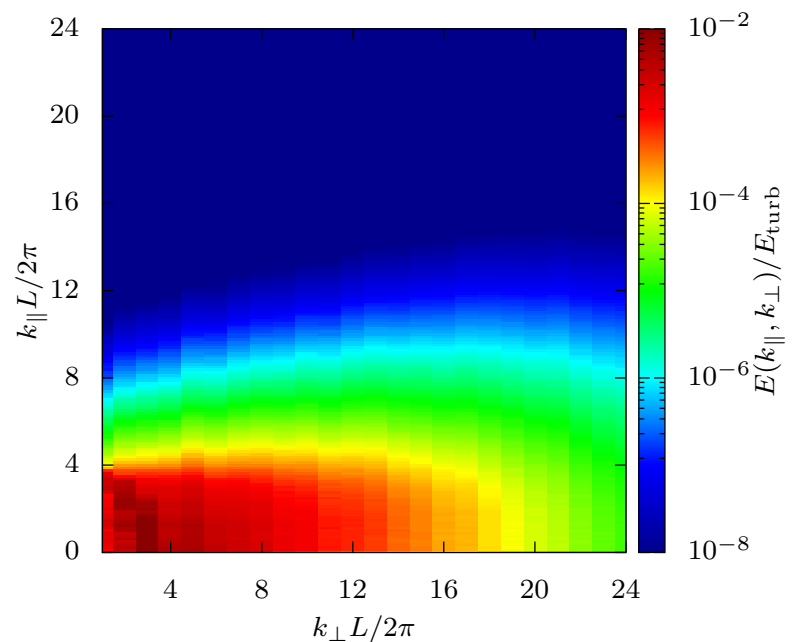

8L-Ms0.02-Ab, $M_{A}=0.2$

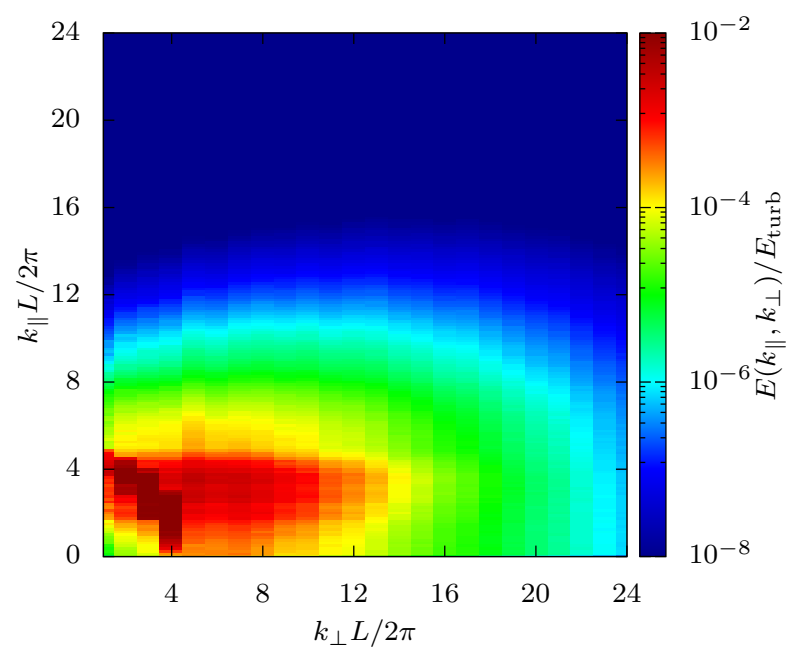

$8 \mathrm{~L}-\mathrm{Ms} 0.02-\mathrm{A}, M_{A}=0.2$

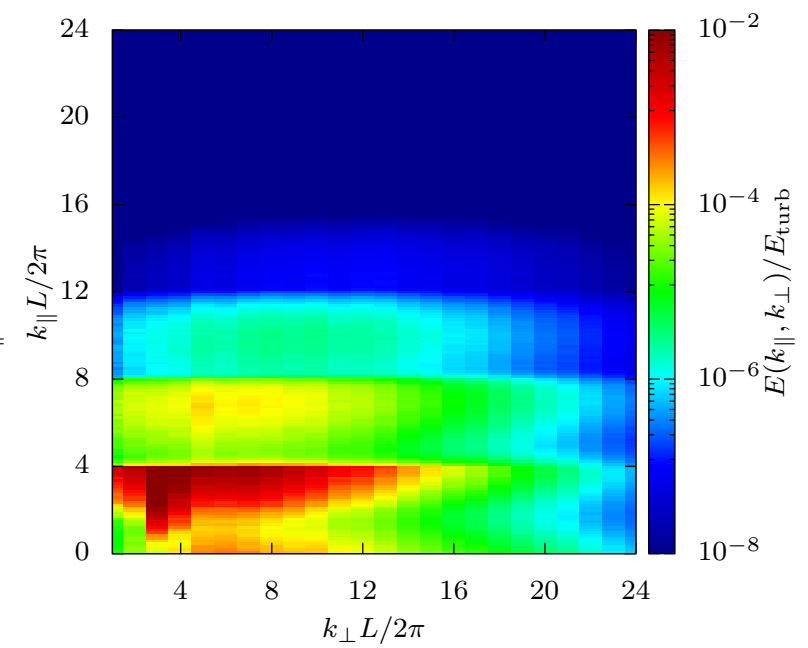

Figure 11. The 2D energy spectrum $E\left(k_{\|}, k_{\perp}\right)$ distribution in the $\left(k_{\|}, k_{\perp}\right)$ plane for models with different turbulence forcing schemes. All the runs have the same sonic Mach number $M_{S}=0.02$ and domain size 8Lx1L. Each column corresponds to a different nominal Alfvénic Mach number $M_{A, 0} \equiv v_{0} / v_{A, 0}$. Left column: $M_{A, 0}=0.4$. Right column: $M_{A, 0}=0.2$. From top to bottom: I-forcing, Ab-forcing, and A-forcing. See Table 1 for the complete description of the simulations parameters. 

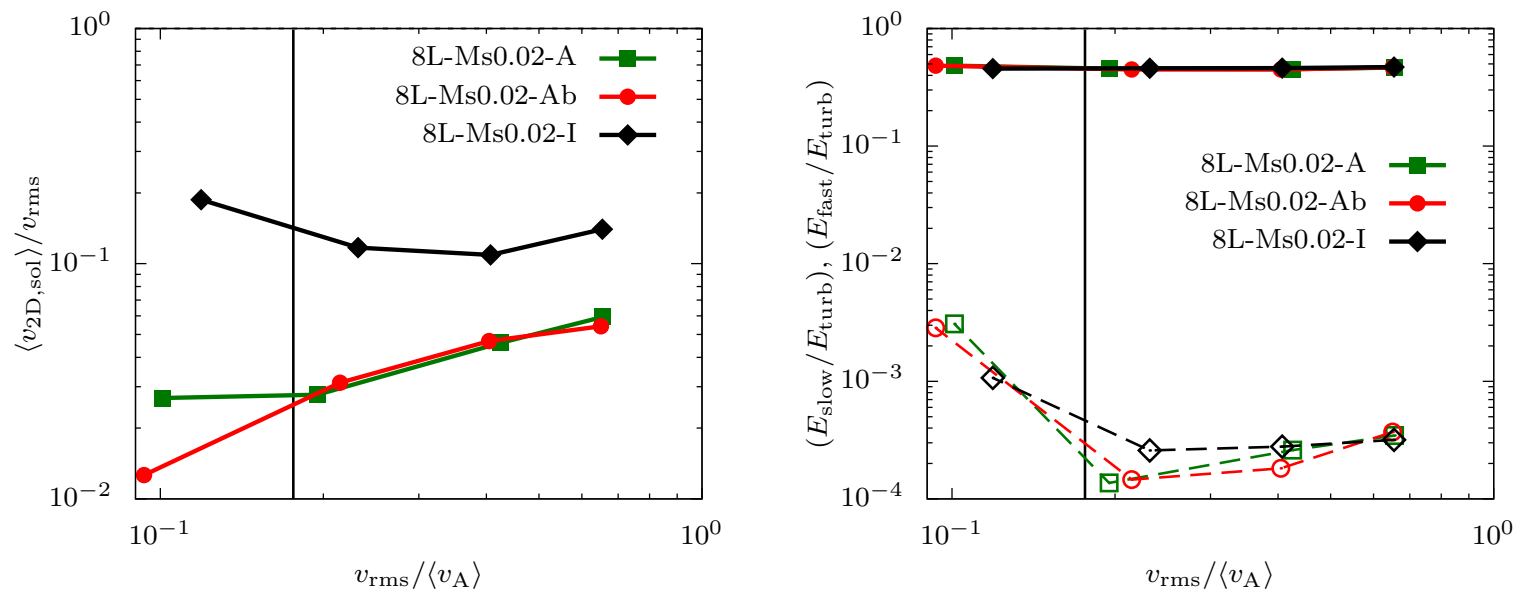

Figure 12. Ratio between the rms value of the $2 \mathrm{D}$ component of the solenoidal velocity $\left\langle v_{2 \mathrm{D} \text {,sol }}\right\rangle$ and the total rms velocity $v_{\text {rms }}$ (left), and the ratio between the energy in each magnetosonic component of the turbulence and the total turbulent energy $E_{\text {turb }}$ (right), as a function of the Alfvénic Mach number $M_{A}=v_{\mathrm{rms}} /\left\langle v_{A}\right\rangle$. In the right panel, the continuous lines are for the energy in the slow modes $E_{\text {slow }}$, and the dashed lines are for the energy in the fast modes $E_{\text {fast }}$. Models with different turbulence forcing schemes are compared in each panel. All the runs have the same sonic Mach number $M_{S}=0.02$ and domain size 8Lx1L. Each point corresponds to a model in Table 1. For the parameters used in these simulations the vertical solid line indicates the lower limit of $M_{A}$ given by Eq. 11 at the injection scale $\ell$.

limit of $M_{A}$ given by Eq. 11 at the injection scale $\ell$. This extension shows more clearly the asymptotic change of the power-law dependence with $M_{A}$ from $\eta_{\text {tf }} / \eta_{\text {hyd }} \sim M_{A}^{3}$ to $\eta_{\mathrm{tf}} / \eta_{\mathrm{hyd}} \sim M_{A}^{2}$, as seen in the models with standard resolution of this work (see Figures 3 and 4).

The top right panel of Fig. 13 compares the normalized energy transfer time from the injection scale, $\tau_{\text {ener }}$, for these simulation sets (see also right panel of Figure 3). We observe that the curve for the set of simulations with lower resolution is slightly steeper, with the power law index in $M_{A}$ between -2 and -3 (for $M_{A}$ larger than some $M_{A}^{*}$, below which the curves become almost flat). This difference of power laws in $\tau_{\text {ener }}$ probably reflects the almost imperceptible steeper power law in $\eta_{\mathrm{tf}}$ for the lower resolution model (top left panel). The break in the curve showing the change in the dependence of $\tau_{\text {ener }}$ with $M_{A}$ (at $M_{A}^{*}$ ) is not evident in the runs set employing standard viscosity and resistivity.

Some small differences between the compared models are also visible in the lower left panel of Figure 13, which depicts the relation between $\left\langle v_{2 \mathrm{D} \text {, sol }}\right\rangle / v_{\text {rms }}$ and $M_{A}$ (see also Figure 7). The higher resolution models have relatively more energy in the $2 \mathrm{D}$ modes (although the maximum difference is still less than a factor of 2). Interestingly, when comparing the energies in the magnetosonic modes for the two resolutions (bottom right panel of Figure 13), we notice that the lower resolution runs show the same increase in the energy of the fast modes seen in the medium resolution ones for the smallest values of $M_{A}$. Nonetheless, this increase happens for higher values of $M_{A}$ in the lower resolution run, contrary to the reduction in the $2 \mathrm{D}$ solenoidal velocity fields.

All the comparisons in Figure 13 suggest that the results for the simulations with the standard resolution (models 16L-Ms0.02-A, having the same cells-size employed in all the simulations presented before in this section) may be close to convergence with respect to the numerical resolution, at least for $\eta_{\mathrm{tf}}$ in the interval of $M_{A}$ considered. Also, the results discussed in the previous sections seem not to be sensitive to changes in the domain size in the direction perpendicular to the mean magnetic field or to the use of the microscopic hyper-diffusivities instead of the standard dissipative terms.

We should also mention that the microscopic resistivity employed in the induction equation of the test-fields (see Appendix A) is the standard resistivity instead of the hyperresistivity employed in the main induction equation. The test-field magnetic diffusivity was kept fixed for all our simulations $\eta_{1}=1.2 \times 10^{-2} \ell_{\perp} v_{0}$ (a value relatively high in order to keep the test-fields smooth for longer times). As discussed in the beginning of this section, we do not expect the microscopic terms to influence the diffusion rate of the large scale magnetic field. Nonetheless, in order to verify if this inconsistency between the microscopic resistive terms could influence the measurement of the turbulent magnetic diffusivity $\eta_{\mathrm{tf}}$, we repeated the run 16L-Ms0.02-low-A, $M_{A}=0.4$, using hyper-resisitivity for the test-fields. The result for $\eta_{\mathrm{tf}}$ (not shown here) revealed no difference.

\section{DISCUSSION}

The diffusion coefficient predicted by the reconnection diffusion (RD) theory in the sub-Alfvénic regime has been derived for the scenario of purely Alfvénic turbulence (i.e., the incompressible limit) in the weak regime. In addition, the scaling laws of the inertial range are assumed to be valid also at the injection scale, as the largest scale motions are responsible for the diffusion rate. The analysis of our simulations does not evince, entirely, the development 

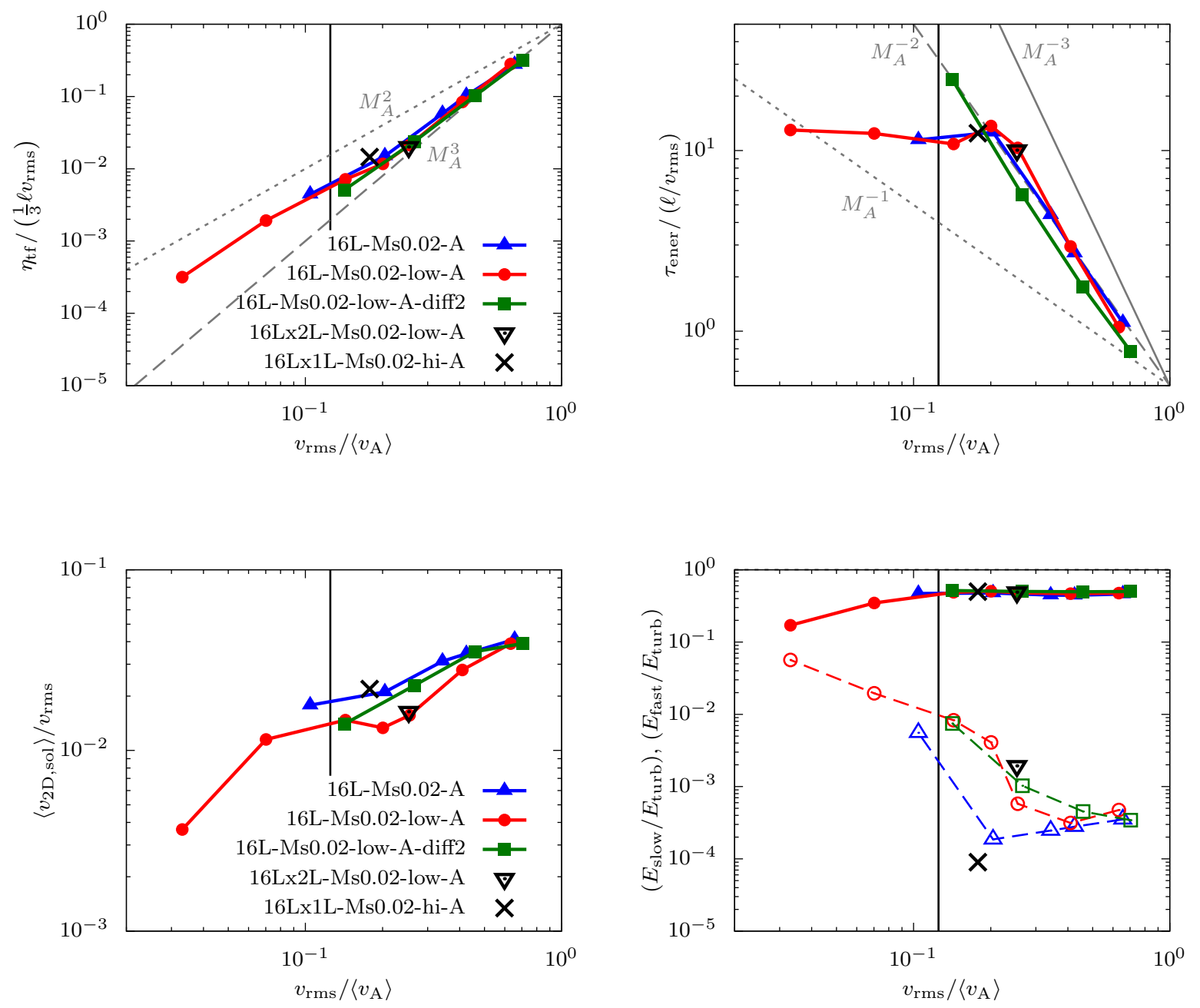

Figure 13. Comparison between models with different numerical resolution (16L-Ms0.02-A, 16L-Ms0.02-low-A, 16L-Ms0.02-hi-A). In addition, models 16L-Ms0.02-low-A are compared with identical counterpart models differing only by the length of the domain perpendicular to the uniform magnetic field (16x2L-Ms0.02-low-A), or by the replacement of the hyper-viscosity and hyper-resistivity by the standard viscosity and resistivity (16L-Ms0.02-low-A-diff2). The plots show different quantities versus the Alfvénic Mach number $M_{A}=v_{\mathrm{rms}} /\left\langle v_{A}\right\rangle$. Top left: magnetic diffusion coefficients $\eta_{\mathrm{tf}}$ measured by the test-field method. Top right: energy transfer time $\tau_{\mathrm{ener}} \equiv E_{\mathrm{turb}} / T_{\mathrm{turb}}$. Bottom left: ratio between the rms value of the $2 \mathrm{D}$ component of the solenoidal velocity $\left\langle v_{2 \mathrm{D} \text {,sol }}\right\rangle$ and the total rms velocity $v_{\mathrm{rms}}$. Bottom right: ratio between the energy in each magnetosonic component of the turbulence and the total turbulent energy $E_{\text {turb. The continuous }}$ lines are for the energy in the slow modes $E_{\text {slow }}$, and the dashed lines are for the energy in the fast modes $E_{\text {fast }}$. All the runs have the same sonic Mach number $M_{S}=0.02$ and domain size 16Lx1L. Each point corresponds to a model in Table 1. For the parameters used in these simulations the vertical solid line indicates the lower limit of $M_{A}$ given by Eq. 11 at the injection scale $\ell$.

of weak turbulence. The resolution available does not allow the analysis of the power law index of the turbulence spectrum, usually employed to characterize the turbulence. However, our estimate of the energy transfer rate at the injection scales shows a dependence $\propto M_{A}^{-\alpha}$ converging to $\alpha=2-3$, which is much stronger than what is expected by the weak turbulence theory (WTT) at the inertial range, $\alpha=1$. It should be pointed out that, to our knowledge there are no results in the literature showing a simulation of forced MHD turbulence reproducing clearly the results of the WTT. However, Meyrand, Kiyani \& Galtier (2014) (see also Meyrand, et al. 2018) identified the WT regime at scales well below the outer scale in a simulation of decaying turbu- lence. Employing a spectral MHD code, Perez \& Boldyrev (2008) performed simulations of forced reduced MHD turbulence and observed a perpendicular power spectrum consistent with the WTT when the injection is performed in a broad range of parallel wave-numbers, although far from the scenario of isotropic injection. From the observational side there is no clear evidence of the MHD WT until the date. The only observation that could be consistent with the WTT is the power spectrum of one of the magnetic field components in the Jupiter's magnetosphere (see Saur, et al. 2002).

Despite of the lack of numerical simulations of forced MHD turbulence demonstrating the development of the WT 
for the cascade immediately from the isotropic injection scale and below, there are several studies (for example Alexakis 2011, 2012; Bigot \& Galtier 2011) exploring forced turbulence in the sub-Alfvénic regime, with isotropic forcing (i.e., strong turbulence is not imposed by anisotropic forcing). These studies show the effects of limited box sizes, such as the development of a 2D dominated cascade, inverse cascade, and the influence of different types of forcing (selection of modes in the $\mathbf{k}$-space), but none of them was able to reproduce the WT power spectrum.

In summary, although the WTT for Alfvén waves is relatively well founded, it seems challenging to produce simulations of forced MHD turbulence in this regime in order to test theories based on the WT scenario (as for example the predictions related to turbulent reconnection, $\mathrm{RD}$, and cosmic ray diffusion). Besides, a robust observational evidence of the MHD WT in nature is still missing.

The simulations presented in this study are not incompressible. However, we kept the induced turbulence subsonic and, although the dominance of the Alfvén waves in the energy spectrum, some level of magneto-sonic waves is seen. The compressible code employed (the Pencil Code) has the advantage to have implemented the test-field method tested in a large number of studies to measure with precision the turbulent diffusion coefficient of the magnetic field. Although the scenario of the RD theory in purely incompressible, some degree of compressibility is expected in the turbulence in astrophysical environments (as for example, in star forming regions of molecular clouds). There is still no theory of weak turbulence for a compressible MHD gas. The cascade of fast modes is weak ${ }^{2}$ at least in subsonic turbulence (see Cho \& Lazarian 2002). Slow modes only cascade fast in the presence of strong Alfvénic turbulence. In the future, it is important to confirm our conclusions for the validity of the RD diffusivity prediction in the asymptotic incompressible limit using for example a spectral MHD code.

In view of all the points raised above, we cannot discard that the turbulence regime developed in our simulations is intermediary between the asymptotic weak and strong limits. This issue can only be investigated by further analysis of simulations with improved resolution. At present, our simulations do not have a clearly identifiable inertial range, although our analysis of the energy transfer spectrum indicates that the numerical dissipation at the injection scales (where the magnetic diffusivity is produced) operates at a rate lower than the cascade/decorrelation rate.

It is therefore surprising that, despite the turbulence statistics of our simulations cannot be directly related to the scenario of the RD theory, we still obtain results close to the predictions (at least when we consider the setups more suitable for the development of the WT).

It is also clear that compressibility modifies the dependence of the diffusion with $M_{A}$, making the diffusion rate closer to the dependence expected when turbulence is in the

2 Weak here means that the interactions decrease with the amplitude of the waves, i.e. $\sim v_{\ell} / \sqrt{c_{s}^{2}+V_{A}^{2}}$, where $c_{s}$ is the sound velocity. The corresponding spectrum corresponds to $\sim k^{-3 / 2}$. The simulations in Kowal \& Lazarian (2010) show that the power spectrum of fast modes can be shock-like, i.e. $\sim k^{-2}$. The latter result still requires further studies with other codes. strong regime (i.e., when the critical balance $\ell_{\|} / v_{a} \sim \ell / \delta u_{\ell}$ is satisfied), and we have $\tau_{\text {casc }} \sim\left(\ell / \delta u_{\ell}\right)$ in eq. (6). In this situation the RD prediction would modify eq. (7) to $\eta_{\mathrm{RD}} \sim D_{\perp} \sim \ell \delta u_{\ell} \min \left(1, M_{A}^{2}\right)$. However, because the amplitude of the $2 \mathrm{D}$ modes of the velocity field increases with the sonic Mach number, it was not possible to disentangle their effects. It remains to explore the turbulent diffusivity in the presence of turbulence more strongly compressible, which is more realistic, e.g., for the interstellar medium. It could be performed by implementing the test-field method in a compressible, shock-capturing MHD code.

Two different forcing schemes for the anisotropic amplitude distribution produced rather similar results. Therefore, the results are apparently not very sensitive to the details of the "k-anisotropic" forcing, provided that the amplitude of the forced modes decreases to zero when the parallel wavenumber goes to zero. One way to strengthen this conclusion in the future would be to repeat this study using a different scheme for driving the turbulence. One particularly interesting scheme consists in inducing random distributions of finite eddies directly in real space. This method was introduced in Kowal, et al. (2012), where it was shown to produce the same turbulent reconnection rates as numerical simulations with forcing controled by their Fourier components (as is done in the present work).

Our results show that the power in the two-dimensional modes, which can eventually dominate the turbulence cascade or dominate the diffusion of the magnetic field, are only controlled when using anisotropic (in $\mathbf{k}$-space) forcing schemes with domains sufficiently elongated in the direction parallel to the mean magnetic field. These results are in agreement with the theoretical limits presented in Nazarenko (2007).

A systematic study of the turbulent diffusivity for subAlfvénic turbulence with a mean magnetic field has been carried out in Karak, et al. (2014, K14). This work also employed the Pencil CODE with the test-field method to measure the diffusion coefficients. One of the setups studied in K14 uses non-helical random forcing, exciting modes isotropically in the $\mathbf{k}$-space. With a square domain and values extremely low of $M_{A}$, the turbulent regime in their simulations could, at least theoretically, be in the discrete WT regime and enslaved to the $2 \mathrm{D}$ cascade (Nazarenko 2007; see Eqs. 11-12). The dependence of the diffusion coefficient with $M_{A}$ observed in K14 (described as a quenching) is close to $M_{A}^{-1}$ for $0.05 \lesssim M_{A} \lesssim 1$, i.e., similar to that we observed in our simulations with isotropic forcing. For the smallest values of $M_{A}$, the dependence with of $\eta_{\text {turb }}$ with $M_{A}$ disappears, which is expected when the diffusion is dominated by the $2 \mathrm{D}$ velocity modes. We can interpret the results of these authors as effects of the simulation setup, specially the domain size.

In the context of numerical simulations of turbulent reconnection, the increase of the reconnection rate could also arise as an artifact of the limited domain size. According to the discussion in the previous paragraphs, in order to avoid this effect, the domain size in the direction parallel to the large-scale magnetic field has to be large enough in these numerical studies.

The present study also calls for a re-evaluation of the transport coefficients in the large scale dynamo context (for example for helical turbulence), taking into account all role 
of compressibility and domain size of the simulation. It is not clear if the same effects also affect the local simulations aimed to extract the mean field coefficients in the convective layer of the Sun or in accretion disks.

The present study supports the predictions of the RD theory at least in the incompressible limit, but it also points to an increase of the diffusivity rate due to the compressibility of the turbulence, alleviating the strong suppression caused by the mean magnetic field. The RD mechanism has been proposed (Lazarian 2005) and tested successfully (at least qualitatively) to solve problems related to star formation: the magnetic flux problem (Santos-Lima, et al. 2010; Leão, et al. 2013) and the magnetic braking catastrophe (Santos-Lima, de Gouveia Dal Pino \& Lazarian 2012, 2013; González-Casanova, Lazarian \& Santos-Lima 2016). Lazarian, Esquivel \& Crutcher (2012) conclude from observations of molecular cloud cores that the mass-to-flux ratio of super-critical cores, compared to their envelopes, are more consistent with the transport of magnetic flux via $R D$ than ambipolar diffusion (AD). More recent works on simulations of star formation processes, encompassing scales from molecular cloud clumps down to protostellar disks through the use of adaptive mesh, try to disentangle the role of other mechanisms that could solve these problems as well, like the misalignment of the angular momentum of the protostellar disk and the mean magnetic field (Joos, Hennebelle, \& Ciardi 2012; Joos et al. 2013), the interchange instability, and the ambipolar diffusion (see, for example, Hennebelle \& Inutsuka 2019 and references therein). These studies in general do not quantify the turbulent magnetic flux transport (due to the inherent difficult to perform this measurement), and it is far from clear if their resolution is able to represent the $\mathrm{RD}$ in these global simulations. For example, Lam, et al. (2019) concluded that only a combination of turbulence and AD (each one dominating in different phases and/or regions) can allow the formation of rotationally supported protostellar disks which persist for sufficiently long times. We remark, however, that the combination "turbulent ambipolar diffusion" is an inconsistent concept: when there is turbulence, this means that the $\mathrm{AD}$ is subdominant; if $\mathrm{AD}$ is very strong, there is no turbulence (see a more complete discussion in the recent review by Lazarian, et al. 2020). The setting and interpretation of such global simulations depend on a full understanding of the RD mechanism driven by turbulence and the numerical simulation setup effects that can interfere in the RD diffusivity. The present study provides a contribution in this direction. Further study of the RD in the presence of gravity are required as the properties of turbulence can be modified by gravity. In fact, Santos-Lima, et al. (2010) found some evidences that the transport of magnetic flux via RD increases with the intensity of the gravitational potential.

\section{SUMMARY AND CONCLUSIONS}

In this work we tested numerically the dependence of the magnetic diffusion coefficient provided by reconnection induced by turbulence (Reconnection Diffusion, RD) with the Alfvénic Mach number of the turbulence. In all our 3D MHD simulations we imposed an initially uniform magnetic field and focused on the sub-Alfvénic regime. The turbulence is forced isotropically in the space. Although we envision applications of the results, e.g., to studies of the role of the RD during star-formation, inside molecular clouds where turbulence is expected to be trans-sonic or supersonic (Santos-Lima, et al. 2010; Santos-Lima, de Gouveia Dal Pino \& Lazarian 2012, 2013; Leão, et al. 2013; González-Casanova, Lazarian \& Santos-Lima 2016), in this study we have focused only in the subsonic case. The motivation is the direct comparison with the current RD theory, built on the scaling laws provided by the Alfvénic turbulence in the weak regime (for sub-Alfvénic isotropic injection). We employed the Pencil Code with the TestField method to extract the average diffusion coefficient from the simulations.

The RD theory assumes that the inertial range scale laws of the weak turbulence theory can be extended to the injection scales, leading to a diffusion coefficient $\eta$ proportional to the hydrodynamical value multiplied by the third power of $M_{A}$, when $M_{A}<1$. We found no clear evidence of the development of the weak turbulence regime in our numerical simulations. In particular, the cascading time $\propto M_{A}^{-1}$ from the weak turbulence theory at the injection scale is not observed in any of our model sets. Due to limited resolution and the fast increase of the cascading time with the increase of the magnetic field intensity, our simulations do not show appreciable inertial range to allow a robust determination of the power law index of the power spectrum. Nonetheless, the diffusion coefficients we obtain seem to be consistent with the RD prediction $\eta \propto M_{A}^{3}$ when the domain size parallel to the uniform magnetic field is large enough to avoid the finite box size effects (see Nazarenko 2007 in the framework of reduced MHD) and the sonic Mach number small enough $\left(M_{S} \lesssim 0.02\right)$. For smaller boxes and bigger values of $M_{S}$, we observed a dependence of $\eta$ more consistent with $M_{A}^{2}$, which could be the expected dependency in the strong cascading regime.

In the future, we will investigate both the incompressible limit to confirm the validity of the RD theory in a larger interval of $M_{A}$, and also the diffusivity provided by supersonic turbulence which is more realistic for star-forming environments. At the same time, more numerical investigation is necessary for the weak turbulence regime, as it is not yet clearly reproducible in direct simulations of forced turbulence.

Due to the omnipresence of MHD turbulence in astrophysics, the proper understanding of the turbulent diffusivity is of fundamental importance not only in the context of star-formation, but it has also important consequences for the more general reconnection problem, for the large scale turbulent dynamo operating in all scales (stars, accretion disks, galaxies; see for example Xu \& Lazarian 2020 for a recent study on the nonlinear turbulent dynamo in a gravitationally collapsing system which accounts for the RD effects), and for the propagation and acceleration of cosmic rays in astrophysical media.

\section{ACKNOWLEDGEMENTS}

The authors thank A. Brandenburg, J. Cho, G. Eyink, B. Raphaldini, M. Rheinhardt, M. V. del Valle, and S. 
$\mathrm{Xu}$ for very useful discussions and comments. The authors also thank the anonymous referee who helped to improve this work with her/his comments. RSL acknowledges partial support from a grant of the Brazilian Agency FAPESP (2013/15115-8), EMGDP from FAPESP (2013/10559-5) and CNPq (306598/2009-4) grants. AL acknowledges the support by NASA TCAN 144AAG1967 grant and NSF AST 1816234 award. The Flatiron Institute is supported by the Simons Foundation. The numerical simulations presented here were performed in the cluster of the Group of Plasmas and High-Energy Astrophysics (GAPAE), acquired with support from FAPESP (grant 2013/10559-5). This work also made use of the computing facilities of the Laboratory of Astroinformatics (IAG/USP, NAT/Unicsul), whose purchase was made possible also by FAPESP (grant 2009/54006-4).

\section{DATA AVAILABILITY}

The data underlying this article will be shared on reasonable request to the corresponding author.

\section{REFERENCES}

Alexakis A., Bigot B., Politano H., Galtier S., 2007, PhRvE, 76, 056313

Alexakis A., 2012, arXiv, arXiv:1209.4225

Alexakis A., 2011, PhRvE, 84, 056330

Armstrong J. W., Rickett B. J., Spangler S. R., 1995, ApJ, 443, 209

Ballesteros-Paredes J., Klessen R. S., Mac Low M.-M., VazquezSemadeni E., 2007, prpl.conf, 63

Beresnyak A., 2014, ApJL, 784, L20

Beresnyak A., Lazarian A., 2019, tuma.book

Bigot B., Galtier S., 2011, PhRvE, 83, 026405

Borue V., Orszag S. A., 1995, EL, 29, 687

Brandenburg A., Chatterjee P., Del Sordo F., Hubbard A., Käpylä P. J., Rheinhardt M., 2010, PhST, 142, 014028

Brandenburg A., Rädler K.-H., Schrinner M., 2008, A\&A, 482, 739

Brandenburg A., Subramanian K., 2005, PhR, 417, 1

Bruno R., Carbone V., 2013, LRSP, 10, 2

Chepurnov A., Lazarian A., Stanimirović S., Heiles C., Peek J. E. G., 2010, ApJ, 714, 1398

Chepurnov A., Burkhart B., Lazarian A., Stanimirovic S., 2015, ApJ, 810, 33

Chepurnov A., Lazarian A., 2010, ApJ, 710, 853

Chepurnov A. V., 1998, A\&AT, 17, 281

Cho J., Lazarian A., Honein A., Knaepen B., Kassinos S., Moin P., 2003, ApJL, 589, L77

Cho J., Lazarian A., Vishniac E. T., 2002, ApJ, 564, 291

Cho J., Lazarian A., 2002, ApJL, 575, L63

Cho J., Lazarian A., 2010, ApJ, 720, 1181

Cho J., Lazarian A., 2003, MNRAS, 345, 325

Cho J., Lazarian A., 2002, PhRvL, 88, 245001

Cho J., Vishniac E. T., 2000, ApJ, 539, 273

Ciolek G. E., Mouschovias T. C., 1993, ApJ, 418, 774. doi:10.1086/173435

Crutcher R. M., Hakobian N., Troland T. H., 2009, ApJ, 692, 844

Elmegreen B. G., Scalo J., 2004, ARA\&A, 42, 211. doi:10.1146/annurev.astro.41.011802.094859

Eyink G., et al., 2013, Natur, 497, 466

Eyink G. L., Lazarian A., Vishniac E. T., 2011, ApJ, 743, 51

Federrath C., Roman-Duval J., Klessen R. S., Schmidt W., Mac Low M.-M., 2010, A\&A, 512, A81
Federrath C., Klessen R. S., 2013, ApJ, 763, 51. doi:10.1088/0004$637 \mathrm{X} / 763 / 1 / 51$

Federrath C., Klessen R. S., 2012, ApJ, 761, 156. doi:10.1088/0004-637X/761/2/156

Galtier S., Nazarenko S. V., Newell A. C., Pouquet A., 2000, JPlPh, 63, 447

Ghosh S., Thomson D. J., Matthaeus W. H., Lanzerotti L. J., 2009, JGRA, 114, A08106. doi:10.1029/2009JA014092

Goldreich P., Sridhar S., 1995, ApJ, 438, 763

Goldstein B. E., Smith E. J., Balogh A., Horbury T. S., Goldstein M. L., Roberts D. A., 1995, GeoRL, 22, 3393

González-Casanova D. F., Lazarian A., Santos-Lima R., 2016, ApJ, 819, 96

Guillet V., Hennebelle P., Pineau des Forêts G., Marcowith A., Commerçon B., Marchand P., 2020, A\&A, 643, A17. doi:10.1051/0004-6361/201937387

Haugen N. E. L., Brandenburg A., 2004, PhRvE, 70, 026405

Haverkorn M., Brown J. C., Gaensler B. M., McClure-Griffiths N. M., 2008, ApJ, 680, 362

Hennebelle P., Chabrier G., 2011, ApJL, 743, L29. doi:10.1088/2041-8205/743/2/L29

Hennebelle P., Inutsuka S.-. ichiro ., 2019, FrASS, 6, 5

Higdon J. C., 1984, ApJ, 285, 109

Hull C. L. H., et al., 2017, ApJL, 842, L9

Iroshnikov P. S., 1963, AZh, 40, 742

Joos M., Hennebelle P., Ciardi A., 2012, A\&A, 543, A128. doi:10.1051/0004-6361/201118730

Joos M., Hennebelle P., Ciardi A., Fromang S., 2013, A\&A, 554, A17. doi:10.1051/0004-6361/201220649

Kandel D., Lazarian A., Pogosyan D., 2017, MNRAS, 470, 3103

Karak B. B., Rheinhardt M., Brandenburg A., Käpylä P. J., Käpylä M. J., 2014, ApJ, 795, 16

Kowal G., Lazarian A., Vishniac E. T., Otmianowska-Mazur K., 2012, NPGeo, 19, 297

Kowal G., Lazarian A., 2010, ApJ, 720, 742

Kowal G., Lazarian A., Vishniac E. T., Otmianowska-Mazur K., 2009, ApJ, 700, 63

Kowal G., Falceta-Gonçalves D. A., Lazarian A., Vishniac E. T., 2017, ApJ, 838, 91

Kowal G., Falceta-Gonçalves D. A., Lazarian A., Vishniac E. T., 2020, ApJ, 892, 50

Kraichnan R. H., 1965, PhFl, 8, 1385

Krasnopolsky R., Li Z.-Y., Shang H., 2010, ApJ, 716, 1541

Krasnopolsky R., Li Z.-Y., Shang H., 2011, ApJ, 733, 54

Krumholz M. R., McKee C. F., 2005, ApJ, 630, 250. doi:10.1086/431734

Lam K. H., Li Z.-Y., Chen C.-Y., Tomida K., Zhao B., 2019, MNRAS, 489, 5326

Larson R. B., 1981, MNRAS, 194, 809

Lazarian A., 2011, arXiv, arXiv:1111.0694

Lazarian A., 2006, ApJL, 645, L25

Lazarian A., 2005, AIPC, 784, 42, AIPC..784

Lazarian A., Esquivel A., Crutcher R., 2012, ApJ, 757, 154

Lazarian A., Pogosyan D., 2000, ApJ, 537, 720

Lazarian A., 2009, SSRv, 143, 357

Lazarian A., Vishniac E. T., 1999, ApJ, 517, 700

Lazarian A., Eyink G. L., Vishniac E. T., Kowal G., 2015, ASSL, 407, 311, ASSL..407

Lazarian A., Eyink G. L., Jafari A., Kowal G., Li H., Xu S., Vishniac E. T., 2020, PhPl, 27, 012305

Leão M. R. M., de Gouveia Dal Pino E. M., Santos-Lima R., Lazarian A., 2013, ApJ, 777, 46

Li Z.-Y., Krasnopolsky R., Shang H., 2011, ApJ, 738, 180

Lithwick Y., Goldreich P., 2001, ApJ, 562, 279

Mac Low M.-M., Klessen R. S., 2004, RvMP, 76, 125

Machida M. N., Inutsuka S.-. ichiro ., Matsumoto T., 2007, ApJ, 670,1198 
Machida M. N., Inutsuka S.-. ichiro ., Matsumoto T., 2009, ApJL, 704, L10

Marchand P., Tomida K., Tanaka K. E. I., Commerçon B., Chabrier G., 2020, ApJ, 900, 180. doi:10.3847/1538$4357 /$ abad99

Maron J., Goldreich P., 2001, ApJ, 554, 1175

Matthaeus W. H., Montgomery D. C., Goldstein M. L., 1983, PhRvL, 51, 1484

McKee C. F., Ostriker E. C., 2007, ARA\&A, 45, 565

Meyrand R., Kiyani K. H., Gürcan Ö. D., Galtier S., 2018, PhRvX, 8, 031066

Meyrand R., Kiyani K. H., Galtier S., 2014, arXiv, arXiv:1409.2092

Montgomery D., Turner L., 1981, PhFl, 24, 825

Myers A. T., McKee C. F., Cunningham A. J., Klein R. I., Krumholz M. R., 2013, ApJ, 766, 97

Nazarenko S., 2007, NJPh, 9, 307

Nishi R., Nakano T., Umebayashi T., 1991, ApJ, 368, 181. doi:10.1086/169682

Padoan P., Juvela M., Kritsuk A., Norman M. L., 2009, ApJL, 707, L153

Padoan P., Nordlund Å., 2011, ApJ, 730, 40. doi:10.1088/0004$637 \mathrm{X} / 730 / 1 / 40$

Perez J. C., Boldyrev S., 2008, ApJL, 672, L61

Santos-Lima R., Lazarian A., de Gouveia Dal Pino E. M., Cho J., 2010, ApJ, 714, 442

Santos-Lima R., de Gouveia Dal Pino E. M., Lazarian A., 2013, MNRAS, 429, 3371

Santos-Lima R., de Gouveia Dal Pino E. M., Lazarian A., 2012, ApJ, 747, 21

Saur J., Politano H., Pouquet A., Matthaeus W. H., 2002, A\&A, 386,699

Schrinner M., Rädler K.-H., Schmitt D., Rheinhardt M., Christensen U., 2005, AN, 326, 245

Schrinner M., Rädler K.-H., Schmitt D., Rheinhardt M., Christensen U. R., 2007, GApFD, 101, 81

Shebalin J. V., Matthaeus W. H., Montgomery D., 1983, JPIPh, 29,525

Shu F. H., 1983, ApJ, 273, 202. doi:10.1086/161359

Shu F., Najita J., Ostriker E., Wilkin F., Ruden S., Lizano S., 1994, ApJ, 429, 781. doi:10.1086/174363

Shu F. H., Galli D., Lizano S., Cai M., 2006, ApJ, 647, 382

Tassis K., Mouschovias T. C., 2005, ApJ, 618, 769. doi:10.1086/424479

Tu C.-Y., Marsch E., 1995, SSRv, 73, 1

Utomo D., Blitz L., Falgarone E., 2019, ApJ, 871, 17

Wolleben M., et al., 2019, AJ, 158, 44

Wurster J., Li Z.-Y., 2018, FrASS, 5, 39. doi:10.3389/fspas.2018.00039

Xu S., Lazarian A., 2020, ApJ, 899, 115. doi:10.3847/1538$4357 /$ aba7ba

Xu S., Zhang B., 2016, ApJ, 824, 113

Xu S., 2020, MNRAS, 492, 1044. doi:10.1093/mnras/stz3092

Yuen K. H., Hu Y., Lazarian A., Pogosyan D., 2019, arXiv, arXiv: 1904.03173

Zhao B., Caselli P., Li Z.-Y., Krasnopolsky R., Shang H., Lam K. H., 2020, arXiv, arXiv:2009.07820

\section{APPENDIX A: THE TEST-FIELD METHOD}

The test-field method, developed initially for spherical geodynamo simulations (Schrinner, et al. 2005), allows to compute unambiguously the contribution of the small scale on the large scale dynamics. In mean-field analysis this contribution is described by the electromotive force, $\langle\mathcal{E}\rangle=$ $\left\langle\mathbf{u}^{\prime} \times \mathbf{B}^{\prime}\right\rangle$, where the primes denote small-scale fields. Consider that $\boldsymbol{u}^{\prime}$ is the turbulent part of a velocity field, $\mathbf{u}$, in a given simulation, and also a set of test-fields, $\boldsymbol{B}^{T}$ (these fields are independent between themselves and of the magnetic field of the simulation $\boldsymbol{B}$ ), such that the evolution of $\boldsymbol{B}^{\prime}$ may be computed in a set of partial differential equations which depends only on $\mathbf{u}$ and $\boldsymbol{B}^{T}$. Thus, it is possible to compute the electromotive force due to the velocity field as $\left\langle\mathcal{E}^{T}\right\rangle=\left\langle\mathbf{u}^{\prime} \times \mathbf{B}^{T \prime}\right\rangle$. For mean magnetic field varying slowly in space and time, and systems where averages in the $x$ and $y$ directions are meaningful, it is possible to write the electromotive force as

$\left\langle\mathcal{E}_{i}\right\rangle=\alpha_{i j}\left\langle B_{j}\right\rangle-\eta_{i j}\left\langle J_{j}\right\rangle$

with, $i, j=1,2$. Thus, with the use of 4 test fields it is possible to obtain the $4+4$ components of $\alpha$ and $\eta$ (see Brandenburg, Rädler \& Schrinner 2008).

For the simulations presented in this work, without rotation and with non-helical unstratified turbulence, the inductive terms in the electromotive force, $\alpha_{i j}$, must be zero on average. Therefore, only 2 test fields would be sufficient to determine the turbulent diffusivity. Nevertheless, we use 4 test fields to verify the existence of other turbulent effects. These are given by

$$
\begin{gathered}
\overline{\boldsymbol{B}}^{1 c}=B_{0}(\cos k z, 0,0), \quad \overline{\boldsymbol{B}}^{2 c}=B_{0}(0, \cos k z, 0), \\
\overline{\boldsymbol{B}}^{1 s}=B_{0}(\sin k z, 0,0), \quad \overline{\boldsymbol{B}}^{2 s}=B_{0}(0, \sin k z, 0),
\end{gathered}
$$

where $k=k_{z, \mathrm{tf}}=2 \pi / \mathrm{L}_{\perp}$. Thus, the $\alpha$-effect is given by the diagonal components of $\alpha_{i j}, \alpha_{x x}=\alpha_{11}$ and $\alpha_{y y}=\alpha_{22}$, while the turbulent diffusion is given by $\eta_{t}=\frac{1}{2}\left(\eta_{11}+\eta_{22}\right)$. In addition, the turbulent driven advection of the magnetic field, often called turbulent pumping, can be measured by $\gamma=\frac{1}{2}\left(\alpha_{21}-\alpha_{12}\right)$. As expected, all the coefficients but $\eta_{t}$ are consistent with zero.

This paper has been typeset from a $\mathrm{T}_{\mathrm{E}} \mathrm{X} / \mathrm{LAT}_{\mathrm{E}} \mathrm{X}$ file prepared by the author. 